\title{
Fixpoint Logic vs. Infinitary Logic in Finite-Model Theory
}

\author{
Phokion G. Kolaitis * \\ Computer and Information Sciences \\ University of California, Santa Cruz \\ Santa Cruz, CA 95064 \\ kolaitis@cse.ucsc.edu
}

\author{
Moshe Y. Vardi \\ Department of Computer Science \\ Rice University \\ Houston, TX 77251-1892 \\ vardi@cs.rice.edu
}

\begin{abstract}
In recent years several extensions of first-order logic have been investigated in the context of finite-model theory. Fixpoint logic and the infinitary logic $L_{\infty \omega}^{\omega}$ with a finite number of variables have turned out to be of particular importance. The study of fixpoint logic generated interactions with both database theory and complexity theory, while the infinitary logic $L_{\infty}^{\omega}$ proved to be a useful tool for analyzing the expressive power of fixpoint logic. In addition to being a proper extension of fixpoint logic, $L_{\infty \omega}^{\omega}$ enjoys a game-theoretic characterization and possesses interesting structural properties, such as the 0-1 law.

In this paper we pursue further the study of the relationship between $L_{\infty}^{\omega}$ and fixpoint logic. We observe that equivalence of two finite structures with respect to $L_{\infty \omega}^{\omega}$ is expressible in fixpoint logic. As a first application of this, we obtain a normal-form theorem for $L_{\infty \omega}^{\omega}$ on finite structures. We then focus on the relative expressive power of first-order logic, fixpoint logic, and $L_{\infty \omega}^{\omega}$ on arbitrary classes of finite structures. Our second main result characterizes when $L_{\infty \omega}^{\omega}$ collapses to first-order logic on an arbitrary class of finite structures. This resolves affirmatively a conjecture of G.L. McColm.
\end{abstract}

\section{Introduction}

In recent years the model theory of finite structures has been a meeting point for research in computer science, combinatorics, and mathematical logic. Results and techniques from finitemodel theory have found interesting applications to several other areas, including database theory [CH82, Var82] and complexity theory [Ajt83, Gur84, Imm86].

One of the distinguishing features of finite-model theory is the fact that first-order logic has severely limited expressive power on finite structures (cf. [Fag75, AU79, Gai82]). In view of that fact, there is an emphasis in finite-model theory on logics that go beyond first-order logic. One way to increase the limited expressive power of first-order logic is by adding fixpoint constructs. Such extensions of first-order logic have been the subject of extensive study - focusing on their

\footnotetext{
${ }^{*}$ During the preparation of this paper this author was partially supported by NSF Grant CCR-9108631
} 
expressive power, their relationship to complexity classes, in particular to $\mathrm{P}$ and PSPACE, and their asymptotic probability properties (cf. [AV89, AV91, Cha88, CH82, Imm86, KV87, Var82]). Of particular importance among these extensions is fixpoint logic, which is obtained from firstorder logic by adding least fixpoints of positive first-order formulas.

Since first-order logic has a finitary syntax, a different way to increase its expressive power is to allow for infinitary formation rules. One of the most powerful logics resulting this way is the infinitary logic $L_{\infty}$ which allows for arbitrary disjunctions and conjunctions. This logic has been studied extensively on infinite structure (cf. [BF85]), but it is too powerful to be of interest or use in finite-model theory, because every class of finite structures that is closed under isomorphisms is definable in $L_{\infty \omega}$.

A more interesting extension from the perspective of finite-model theory is the infinitary logic $L_{\infty \omega}^{\omega}$, which consists of all formulas of $L_{\infty}$ with a finite number of distinct variables. More formally, $L_{\infty \omega}^{\omega}$ is the union $\bigcup_{k=1}^{\infty} L_{\infty \omega \omega}^{k}$, where $L_{\infty \omega}^{k}$ is the collection of all formulas of $L_{\infty \omega}$ with $k$ variables. The infinitary logic $L_{\infty \omega}^{\omega}$ was studied originally on infinite structures [Bar77], but it turned out to have numerous uses in finite-model theory. Indeed, $L_{\infty \omega}^{\omega}$ could be viewed as underlying much of the work on lower bounds for expressibility on finite structures in [Imm82, dR87, LM89, CFI89], although its use there is rather implicit. Moreover, in [KV90a, KV90b], the infinitary logic $L_{\infty \omega}^{\omega}$ was studied systematically in its own right and the importance of it on finite structures became evident.

One of the reasons for the importance of $L_{\infty}^{\omega}$ in finite-model theory is the fact that from the expressive power standpoint $L_{\infty \omega}^{\omega}$ constitutes a proper extension of fixpoint logic [KV90b]. Thus, $L_{\infty \omega}^{\omega}$ can be used to derive both positive and negative results about fixpoint logic. On the positive side, structural results about $L_{\infty \omega}^{\omega}$ transfer to similar results for fixpoint logic, while, on the negative side, lower-bound results for expressibility in $L_{\infty \omega}^{\omega}$ yield, a fortiori, lower bounds for expressibility in fixpoint logic (but not vice-versa). The main advantage of $L_{\infty \omega}^{\omega}$ is that the expressive power of the infinitary $\operatorname{logics} L_{\infty \omega}^{k}$ with $k$ variables, $k \geq 1$, has a clean and precise characterization in terms of certain $k$-pebble games between two players on a pair of structures (cf. [Bar77, Imm82, KV90b]). In contrast, fixpoint logic is not known to possess a similar property. This game-theoretic characterization of $L_{\infty \omega}^{\omega}$ can be used to establish the 0-1 law for the asymptotic probabilities of properties expressible in $L_{\infty \omega}^{\omega}$ [KV90b]. Moreover, the pebble games for $L_{\infty \omega}^{\omega}$ have been the most fruitful technique developed so far in establishing lower-bound results for expressibility in fixpoint logic (cf. [Imm82, dR87, LM89, CFI89, KV90a]).

In this paper we continue the systematic investigation of the infinitary logic $L_{\infty \omega}^{\omega}$ on finite structures. Our goal is to obtain a better insight into the expressive power of $L_{\infty \omega}^{\omega}$ on arbitrary classes of finite structures and to illuminate the relationship and interaction between $L_{\infty \omega}^{\omega}$ and fixpoint logic.

We begin by bringing into center stage a basic fact about $L_{\infty \omega}^{\omega}$ and fixpoint logic, which has been implicit in [IL90, KV90b, AV91]. In a nutshell, this fact asserts that fixpoint logic can capture equivalence in the infinitary logics $L_{\infty \omega}^{k}$ with $k$ variables, $k \geq 1$. More precisely, for each $k \geq 1$ the query "given two finite structures $\mathbf{A}$ and $\mathbf{B}$, do they satisfy the same sentences of $L_{\infty \omega}^{k}$ ?" is expressible in fixpoint logic. At first sight this appears to be rather counter-intuitive, since fixpoint logic is only a very small fragment of $L_{\infty \omega}^{\omega}$, but it becomes more plausible when one thinks of the game-theoretic characterization of $L_{\infty \omega}^{k}$ and realizes that the above query is equivalent to asking who of the two players wins the $k$-pebble game on $\mathbf{A}$ and $\mathbf{B}$. 
In this paper we turn the above fact into a technical tool for studying $L_{\infty}^{\omega}$ and we obtain two main results. The first result is a normal-form theorem for $L_{\infty}^{\omega}$ that sheds light into the difference between fixpoint logic and $L_{\infty}^{\omega}$. In general, formulas of $L_{\infty}^{\omega}$ are constructed from atomic formulas by applying repeatedly negation, quantification, and infinitary conjunction and disjunction (subject to the restriction on the number of variables). Thus, at the level of the syntax a formula of $L_{\infty \omega}^{\omega}$ may involve any finite number of alternations between infinitary disjunctions and infinitary conjunctions. We prove here, however, that every formula of $L_{\infty \omega}^{\omega}$ is semantically equivalent to a countable disjunction of first-order formulas with a uniform bound on the number of variables, i.e., the infinitary connective has to be used only once. Since it is known that fixpoint formulas are semantically equivalent to "syntactically regular" countable disjunctions of first-order formulas, our result indicates that the extra power of $L_{\infty \omega}^{\omega}$ is derived solely from the ability to form an arbitrary countable disjunction of first-order formulas.

Our second main result has to do with the comparison between the expressive power of $L_{\infty \omega}^{\omega}$, fixpoint logic, and first-order logic over arbitrary classes of finite structures. Typically, most expressiveness results in finite-model theory are with respect to the class of all finite structures. In practice, however, one is often concerned with a restricted class of finite structures, such as the class of trees, the class of planar graphs, or the class of databases satisfying a certain constraint. Thus, there is a solid motivation in attempting to understand the relationship between $L_{\infty}^{\omega}$, fixpoint logic, and first-order logic on specific classes of finite structures. It should be pointed out that, while these three logics have distinct expressive power over the class of all finite structures, they may have identical expressive power over restricted classes of finite structures. This is, for example, the case with the class of finite equivalence relations, where both fixpoint logic and $L_{\infty}^{\omega}$ collapse to first-order logic (cf. [Kol85]).

Under what conditions does fixpoint logic or $L_{\infty \omega}^{\omega}$ collapse to first-order logic over a given class of finite structures? This problem was raised and studied by McColm [McC90] who formulated two conjectures asserting that the collapse of fixpoint logic or of $L_{\infty \omega}^{\omega}$ down to first-order logic is equivalent to a certain boundedness condition on fixpoint logic.

Let $\mathcal{C}$ be a class of finite structures and let $\varphi^{\infty}$ be the least fixpoint of a positive first-order formula $\varphi(\mathbf{x}, S)$. We say that the formula $\varphi(\mathbf{x}, S)$ is bounded on $\mathcal{C}$ if the least fixpoint $\varphi^{\infty}$ is reached after a bounded number of iterations, i.e., there is a positive integer $N$ such that on every structure in $\mathcal{C}$ the least fixpoint $\varphi^{\infty}$ is reached by iterating $\varphi(\mathbf{x}, S)$ at most $N$ times. This concept occupies a central place in the theory of database queries, where it has been studied in the context of Datalog, a database query language which can be viewed as the existential and negation-free fragment of fixpoint logic (cf. [Ioa86, GMSV87, CGKV88, Nau89, KA89]). Observe that if $\varphi$ is bounded on $\mathcal{C}$, then its least fixpoint $\varphi^{\infty}$ is first-order definable on $\mathcal{C}$, since it is equivalent to the $N$-th iterant of $\varphi$. The converse, however, is not always true. In particular, over the class of total orders there are natural examples of unbounded first-order formulas whose least fixpoints are first-order definable. Ajtai and Gurevich [AG89] have investigated the precise relationship between boundedness and first-order definability for Datalog programs.

If every positive first-order formula $\varphi(\mathbf{x}, S)$ is bounded on a class $\mathcal{C}$ of finite structures, then we say that fixpoint logic is bounded on $\mathcal{C}$. The preceding remarks imply that boundedness of fixpoint logic on $\mathcal{C}$ is a sufficient condition for collapsing fixpoint logic to first-order logic on $\mathcal{C}$. McColm's [McC90] first conjecture asserts that this is the only way that fixpoint can collapse to first-order logic on a class $\mathcal{C}$ of finite structures, i.e., fixpoint logic has the same expressive power 
as first-order logic on $\mathcal{C}$ if and only if fixpoint logic is bounded on $\mathcal{C}$. McColm's [McC90] second conjecture states that boundedness of fixpoint logic is also the key to the collapse of $L_{\infty \omega}^{\omega}$ down to first-order logic. More precisely, the second conjecture asserts that $L_{\infty \omega}^{\omega}$ has the same expressive power as first-order logic on $\mathcal{C}$ if and only if fixpoint logic is bounded on $\mathcal{C}$. Notice that the two conjectures combined together assert that fixpoint logic collapses to first-order logic on a class $\mathcal{C}$ of finite structures if and only if $L_{\infty \omega}^{\omega}$ collapses to first-order logic on $\mathcal{C}$.

What is the intuition behind these two conjectures? Underlying these conjecture is the belief that the ability to go beyond first-order logic has to do with the ability to form nontrivial countable disjunctions of first-order formulas. Moreover, the empirical evidence for these conjectures appears to be strong, since none of the classes $\mathcal{C}$ of finite structures studied so far is known to violate them (cf. [McC90]). For example, on the class of finite equivalence relations fixpoint logic is bounded and $L_{\infty \omega}^{\omega}$ collapses to first-order logic, while on the class of total orders fixpoint logic is unbounded and has strictly higher expressive power than first-order logic.

In this paper, we confirm McColm's second conjecture, i.e., we show that $L_{\infty \omega}^{\omega}$ collapses to first-order logic on an arbitrary class $\mathcal{C}$ of finite structures if and only if fixpoint logic is bounded on $\mathcal{C}$. This result turns out to have an unexpected application to $0-1$ laws, namely, we derive a necessary and sufficient condition for the existence of 0-1 laws for $L_{\infty}^{\omega}$ under variable probability measures on an arbitrary class $\mathcal{C}$ of finite structures.

We also examine McColm's first conjecture and give evidence that a special case of this conjecture might have complexity-theoretic implications. In particular, the first conjecture appears to have a different flavor than the second one and to require the development of new tools.

\section{Infinitary Logics and Fixpoint Logics}

A vocabulary $\sigma$ is a finite set of relation symbols $R_{i}, 1 \leq i \leq s$, and constant symbols $c_{j}, 1 \leq j \leq t$. A structure $\mathbf{A}=\left(A, R_{1}^{\mathbf{A}}, \ldots, R_{s}^{\mathbf{A}}, c_{1}^{\mathbf{A}}, \ldots, c_{t}^{\mathbf{A}}\right)$ over the vocabulary $\sigma$ consists of a set $A$, called the universe of $\mathbf{A}$, relations $R_{i}^{\mathbf{A}} \subseteq A^{r_{i}}$, where $r_{i}$ is the arity of the relation symbol $R_{i}, 1 \leq i \leq s$, and

distinguished elements $c_{j}^{\mathbf{A}}, 1 \leq j \leq t$, from the universe $A$ of $\mathbf{A}$. We say that a structure $\mathbf{A}$ is finite if its universe is a finite set.

If $k$ is a positive integer, then a $k$-ary query $Q$ on $\sigma$ is a mapping that associates a $k$-ary relation $Q(\mathbf{A})$ on $A$ with each finite structure $\mathbf{A}$ over $\sigma$ such that $Q(\mathbf{A})$ is preserved under isomorphisms. In other words, if $\mathbf{A}$ and $\mathbf{B}$ are finite structures over $\sigma$ and $f$ is an isomorphism from $\mathbf{A}$ to $\mathbf{B}$, then $f$ is also an isomorphism from $(A, Q(\mathbf{A}))$ to $(B, Q(\mathbf{B}))$. A Boolean query on $\sigma$ is a mapping $Q$ from finite structures over $\sigma$ to $\{0,1\}$ such that if $\mathbf{A}$ and $\mathbf{B}$ are isomorphic, then $Q(\mathbf{A})=\mathbf{Q}(\mathbf{B})$. Equivalently, a Boolean query can be identified with a class of finite structures over $\sigma$ that is closed under isomorphisms.

The complement of a $k$-ary query $Q$ over $\sigma$ is the $k$-ary query $Q^{*}$ such that $Q^{*}(\mathbf{A})=A^{k}-Q(\mathbf{A})$ for every finite structure $\mathbf{A}$ over $\sigma$. Similary, the complement of a Boolean query $Q$ is the Boolean query $Q^{*}$ such that $Q^{*}(\mathbf{A})=1-Q(\mathbf{A})$ for every finite structure $\mathbf{A}$ over $\sigma$.

Let $\mathcal{L}$ be a logic, $k$ a positive integer, $Q$ a $k$-ary query over the vocabulary $\sigma$, and $\varphi\left(x_{1}, \ldots, x_{k}\right)$ a formula of $\mathcal{L}$ with free variables among $x_{1}, \ldots, x_{k}$. We say that the formula $\varphi\left(x_{1}, \ldots, x_{k}\right)$ defines the query $Q$ if for every finite structure $\mathbf{A}$ over $\sigma$ and every sequence $\left(a_{1}, \ldots, a_{k}\right)$ from the universe $A$ of $\mathbf{A}$ we have that

$$
\left(a_{1}, \ldots a_{k}\right) \in Q(\mathbf{A}) \Longleftrightarrow \mathbf{A} \models \varphi\left(x_{1} / a_{1}, \ldots, x_{k} / a_{k}\right),
$$


where $\mathbf{A} \models \varphi\left(x_{1} / a_{1}, \ldots, x_{k} / a_{k}\right)$ means that the structure $\mathbf{A}$ satisfies the formula $\varphi\left(x_{1}, \ldots, x_{k}\right)$ when the variable $x_{i}$ is interpreted by the element $a_{i}, 1 \leq i \leq k$. We say that the query $Q$ is $\mathcal{L}$-definable (or, equivalently, $Q$ is an $\mathcal{L}$-query) if there is a formula of $\mathcal{L}$ that defines $Q$.

The above definitions can be relativized to a class $\mathcal{C}$ of finite structures that is closed under isomorphisms, so that we have the concepts of a query on $\mathcal{C}$ and and an $\mathcal{L}$-definable query on $\mathcal{C}$. ¿From now on, we make the blanket assumption that the term class $\mathcal{C}$ of finite structures over the vocabulary $\sigma$ means that $\mathcal{C}$ is a class of finite structures over $\sigma$ that is closed under isomorphisms.

\subsection{Infinitary Logics with a Fixed Number of Variables}

The infinitary logic $L_{\infty}$ is the extension of first-order logic that results by allowing infinite disjunctions and conjunctions in the syntax, while keeping the quantifier strings finite (cf. [BF85]). More formally, the syntax of $L_{\infty \omega}$ is obtained by augmenting the syntax of first-order logic with the following rule: If $\Psi$ is a set of formulas of $L_{\infty \omega}$, then the expressions $\bigvee \Psi$ and $\bigwedge \Psi$ are also formulas of $L_{\infty} \omega$. The semantics of infinitary formulas is a direct extension of the semantics of first-order logic, where $\bigvee \Psi$ is interpreted as a disjunction over all formulas in $\Psi$ and $\bigwedge \Psi$ is interpreted as a conjunction. In general, formulas of $L_{\infty \omega}$ may have an infinite number of distinct variables. We now focus attention on fragments of $L_{\infty \omega}$ in which the total number of variables is required to be finite. Variables, however, may have an unbounded number of occurrences in such formulas.

Definition 2.1: Let $k$ be a positive integer.

- The infinitary logic $L_{\infty \omega}^{k}$ with $k$ variables consists of all formulas of $L_{\infty \omega}$ with at most $k$ distinct variables.

- The infinitary logic $L_{\infty}^{\omega}$ with finitely many variables consists of all formulas of $L_{\infty \omega}$ with a finite number of distinct variables, i.e., $L_{\infty \omega}^{\omega}=\bigcup_{k=1}^{\infty} L_{\infty \omega}^{k}$.

- $L_{\omega \omega}^{k}$ is the collection of all first-order formulas with at most $k$ distinct variables.

The expressive power of the $\operatorname{logics} L_{\infty \omega}^{k}, k \geq 1$, is usually illustrated by the fact that for any $n \geq 1$ the property $p_{n}(x, y)$ asserting that "there is a path of length $n$ from $x$ to $y$ " is expressible by a formula of $L_{\omega \omega}^{3}$. Indeed, put $p_{1}(x, y) \equiv E(x, y)$ and assume, by induction on $n$, that $p_{n-1}(x, y)$ is equivalent to a formula of $L_{\omega \omega}^{3}$. Then the desired formula $p_{n}(x, y)$ is

$$
p_{n}(x, y) \equiv(\exists z)\left[E(x, z) \wedge(\exists x)\left(x=z \wedge p_{n-1}(x, y)\right)\right] .
$$

As a result, the formula $(\forall x)(\forall y)\left(\bigvee_{n=1}^{\infty} p_{n}(x, y)\right)$ of $L_{\infty \omega}^{3}$ defines the transitive closure query on graphs. More generally, if $P$ is any set of positive integers, then the property " $x$ and $y$ are connected by a path whose length is a number in $P$ " is expressible in $L_{\infty \omega}^{3}$ via the formula $\bigvee_{n \in P} p_{n}(x, y)$. It follows that $L_{\infty}^{\omega}$ can express non-recursive queries on finite graphs. For additional background information on infinitary logics with a fixed number of variables we refer the reader to $[\mathrm{KV} 92 \mathrm{~b}]$. 


\subsection{Fixpoint Logic and Partial Fixpoint Logic}

Let $\varphi\left(x_{1}, \ldots, x_{n}, S\right)$ be a first-order formula in which $S$ is a new $n$-ary relation symbol (not included in $\sigma$ ), and let $\mathbf{A}$ be a structure over the vocabulary $\sigma$. The formula $\varphi$ gives rise to an operator $\Phi$ from $n$-ary relations on the universe $A$ of $\mathbf{A}$ to $n$-ary relations on $A$, where for every $n$-ary relation $R$ on $A$

$$
\Phi(R)=\left\{\left(a_{1}, \ldots, a_{n}\right): \mathbf{A} \models \varphi\left(x_{1} / a_{1}, \ldots, x_{n} / a_{n}, S / R\right)\right\} .
$$

Every such operator $\Phi$ can be iterated and, thus, it gives rise to the sequence of stages $\Phi^{m}, m \geq 1$, defined by the induction:

$$
\Phi^{1}=\Phi(\emptyset), \Phi^{m+1}=\Phi\left(\Phi^{m}\right)
$$

If the formula $\varphi\left(x_{1}, \ldots, x_{n}, S\right)$ is positive in $S$ (which means that every occurrence of $S$ in $\varphi$ is within an even number of negations), then the associated operator $\Phi$ is monotone, i.e., $\Phi\left(R_{1}\right) \subseteq \Phi\left(R_{2}\right)$, whenever $R_{1} \subseteq R_{2}$. Thus, for positive formulas the sequence $\Phi^{m}, m \geq 1$, of stages is increasing. It follows that if $\mathbf{A}$ is a finite structure and $\varphi\left(x_{1}, \ldots, x_{n}, S\right)$ is a positive in $S$ formula, then there is an integer $m_{0} \leq|A|^{n}$, where $|A|$ is the cardinality of the universe $A$ of A, such that $\Phi^{m_{0}}=\Phi^{m}$ for every $m \geq m_{0}$. Moreover, it is easy to verify that $\Phi^{m_{0}}$ is the least fixpoint of the operator $\Phi$ on the finite structure $\mathbf{A}$, i.e., it is the smallest $n$-ary relation $S$ on $A$ such that $\Phi(S)=S$. We write $\varphi^{\infty}\left(x_{1}, \ldots, x_{n}\right)$ to denote the least fixpoint of $\Phi$ and we refer to it as the least fixpoint of the formula $\varphi\left(x_{1}, \ldots, x_{n}, S\right)$.

Remark 2.2: Although here we are mainly interested in finite structures, we should point out that the stages of a formula can also be defined on infinite structures. This is done by transfinite induction on the ordinals, where at limit stages the operator $\Phi$ is applied to the union of the previously defined stages. A positive formula has a least fixpoint on every infinite structure, which is equal to some transfinite stage of the formula (cf. [Mos74]).

Definition 2.3: Fixpoint logic FP is the extension of first-order logic that contains all first-order formulas and is closed under the positive operations of first-order logic (i.e., finitary disjunctions and conjunctions, existential and universal quantification) and the following least fixpoint rule: If $\varphi\left(x_{1}, \ldots, x_{n}, S\right)$ is a positive first-order formula, then $\varphi^{\infty}\left(x_{1}, \ldots, x_{n}\right)$ is also a formula of FP.

Notice that at the level of the syntax fixpoint logic FP is not closed under negation. On the other hand, Immerman [Imm86] showed that on finite structures the queries expressible by formulas of FP are closed under complements (cf. also [Imm86, GS86]).

The canonical example illustrating the expressive power of fixpoint logic is provided by the least fixpoint $\varphi^{\infty}(x, y)$ of the first-order formula $E(x, y) \vee(\exists z)(S(x, z) \wedge S(z, y))$. In this case, $\varphi^{\infty}(x, y)$ is a formula of fixpoint logic that defines the transitive closure of the edge relation $E$.

If the operator $\Phi$ is not monotone, then on a given finite structure $\mathbf{A}$ either there is a positive integer $m$ such that $\Phi^{m}=\Phi^{m+1}$ or the sequence $\Phi^{m}, m \geq 1$, of stages cycles without ever yielding a fixpoint of $\Phi$, which means that there is an integer $r>1$ such that $\Phi^{m+r}=\Phi^{m}$, but $\Phi^{m+r^{\prime}} \neq \Phi^{m}$ for all positive integers $r^{\prime}<r$. If $\varphi\left(x_{1}, \ldots, x_{n}, S\right)$ is an arbitrary first-order formula, 
we define the partial fixpoint $\varphi^{\infty}\left(x_{1}, \ldots, x_{n}\right)$ of $\varphi$ on a finite structure $\mathbf{A}$ to be a stage $\Phi^{m}$ such that $\Phi^{m}=\Phi^{m+1}$, if such a stage exists, or the empty set $\emptyset$, otherwise. It should be pointed out that the partial fixpoint need not be the least fixpoint, even if the sequence of stages converges to a fixpoint, since, after all, the least fixpoint may not exist.

Definition 2.4: Partial Fixpoint Logic PFP is the extension of first-order logic that contains all first-order formulas and is closed under the operations of first-order logic and the following partial fixpoint rule: if $\varphi\left(x_{1}, \ldots, x_{n}, S\right)$ is an arbitrary first-order formula, then the partial fixpoint $\varphi^{\infty}\left(x_{1}, \ldots, x_{n}\right)$ of $\varphi$ is also a formula of PFP.

Definition 2.5: If $\mathcal{C}$ is a class of finite structures over the vocabulary $\sigma$, then we write $\operatorname{FP}(\mathcal{C})$ and $\operatorname{PFP}(\mathcal{C})$ to denote the classes of queries on $\mathcal{C}$ that are definable in fixpoint logic and partial fixpoint logic, respectively. We refer to these queries as fixpoint queries on $\mathcal{C}$ and partial fixpoint queries on $\mathcal{C}$.

If $\mathcal{C}$ is the class of all finite structures over the vocabulary $\sigma$, then we write simply $\mathrm{FP}$ and PFP to denote the classes of fixpoint queries and partial fixpoint queries on all finite structures, respectively. We trust that the reader will be able to tell from the context if the notation $\mathrm{FP}$ and PFP refers to formulas of fixpoint logic and partial fixpoint logic or to queries definable by formulas of these logics.

The interested reader can find in [KV92b] several remarks about the history and evolution of fixpoint logic and partial fixpoint logic. On the technical side, we now state certain important facts about these logics that will be of interest and use to us here.

Both fixpoint logic and partial fixpoint logic embody recursion through the iteration of single formulas. One can also consider a seemingly more powerful recursion mechanism that allows for the simultaneous iteration of a finite sequence of first-order formulas. A system of first-order formulas is a finite sequence

$$
\varphi_{1}\left(x_{1}, \ldots, x_{n_{1}}, S_{1}, \ldots, S_{l}\right), \ldots, \varphi_{l}\left(x_{1}, \ldots, x_{n_{l}}, S_{1}, \ldots, S_{l}\right)
$$

of first-order formulas such that each $S_{i}$ is a relation symbol of arity $n_{i}, 1 \leq i \leq l$, not in the vocabulary $\sigma$. If $\mathbf{A}$ is a structure over the vocabulary $\sigma$, then every such system gives rise to an operator $\Phi$ from sequences $\left(R_{1}, \ldots, R_{l}\right)$ of relations $R_{i}$ of arity $n_{i}, 1 \leq i \leq l$, on the universe $A$ of A to sequences of relations on $A$ of the same arities. More precisely,

$$
\Phi\left(R_{1}, \ldots, R_{l}\right)=\left(\Phi_{1}\left(R_{1}, \ldots, R_{l}\right), \ldots, \Phi_{l}\left(R_{1}, \ldots, R_{l}\right)\right),
$$

where for every $i \leq l$

$$
\Phi_{i}\left(R_{1}, \ldots, R_{l}\right)=\left\{\left(a_{1}, \ldots, a_{n_{i}}\right): \mathbf{A} \models \varphi_{i}\left(x_{1} / a_{1}, \ldots, x_{n_{i}} / a_{n_{i}}, S_{1} / R_{1}, \ldots, S_{l} / R_{l}\right)\right\} .
$$

The stages $\Phi^{m}=\left(\Phi_{1}^{m}, \ldots, \Phi_{l}^{m}\right), m \geq 1$, of $\Phi$ on a structure $\mathbf{A}$ are defined by the induction:

$$
\Phi^{1}=\Phi(\emptyset, \ldots, \emptyset), \Phi^{m+1}=\Phi\left(\Phi_{1}^{m}, \ldots, \Phi_{l}^{m}\right), m \geq 1 .
$$


If each formula $\varphi_{i}\left(x_{1}, \ldots, x_{n_{i}}, S_{1}, \ldots, S_{l}\right), 1 \leq i \leq l$, of a system is positive in every relation symbol $S_{1}, \ldots, S_{l}$, then the associated operator $\Phi$ is monotone in each of its arguments and, as a result, the sequence of its stages is increasing in each component. Thus, for every finite structure $\mathbf{A}$ the sequence of stages of $\Phi$ converges after finitely many iterations, i.e., there is a positive integer $m_{0}$ such that $\Phi^{m}=\Phi^{m_{0}}$ for every $m \geq m_{0}$. Moreover, the sequence $\Phi^{m_{0}}=\left(\Phi_{1}^{m_{0}}, \ldots, \Phi_{l}^{m_{0}}\right)$ is the least fixpoint of the operator $\Phi$ on $\mathbf{A}$, i.e., the smallest sequence $\left(R_{1}, \ldots, R_{l}\right)$ of relations on $A$ such that $\Phi\left(R_{1}, \ldots, R_{l}\right)=\left(R_{1}, \ldots, R_{l}\right)$. We call this sequence the least fixpoint of the system $\varphi_{1}, \ldots, \varphi_{l}$ and denote it by $\left(\varphi_{1}^{\infty}, \ldots, \varphi_{l}^{\infty}\right)$. Usually, one is interested not in the entire sequence $\left(\varphi_{1}^{\infty}, \ldots, \varphi_{l}^{\infty}\right)$, but in only one of its components, for example, in the last component $\varphi_{l}^{\infty}$. We say that $\varphi_{l}^{\infty}\left(x_{1}, \ldots, x_{n_{l}}\right)$ is the goal of the system $\varphi_{1}\left(x_{1}, \ldots, x_{n_{1}}, S_{1}, \ldots, S_{l}\right), \ldots, \varphi_{l}\left(x_{1}, \ldots, x_{n_{l}}, S_{1}, \ldots, S_{l}\right)$.

To illustrate the above concepts, consider the system

$$
\begin{aligned}
\varphi_{1}\left(x, y, S_{1}, S_{2}\right) & \equiv E(x, y) \vee(\exists z)\left(S_{1}(x, z) \wedge S_{1}(z, y)\right) \\
\varphi_{2}\left(x, S_{1}, S_{2}\right) & \equiv S_{1}(x, x)
\end{aligned}
$$

On every graph $G=(V, E)$, the first component $\varphi_{1}^{\infty}(x, y)$ of the least fixpoint of this system defines the transitive closure query, while the goal $\varphi_{2}^{\infty}(x)$ defines the cycle query, i.e., the query that computes all nodes $a \in V$ such that there is a path from $a$ to $a$.

Consider now first-order logic augmented with the following least fixpoint rule for systems: if $\varphi_{1}, \ldots, \varphi_{l}$ is a system of positive first-order formulas, then the goal $\varphi_{l}^{\infty}\left(x_{1}, \ldots, x_{n_{l}}\right)$ of the system is also a formula. It is well known that this extension of first-order logic coincides with fixpoint logic FP. This is established by showing that a system of first-order formulas can be simulated by a single formula $\varphi\left(x_{1}, \ldots, x_{q}, S\right)$ in which $S$ is a relation symbol of sufficiently large arity $q$, so that the goal $\varphi_{l}^{\infty}\left(x_{1}, \ldots, x_{n_{l}}\right)$ of the system can be defined from the least fixpoint $\varphi^{\infty}\left(x_{1}, \ldots, x_{q}\right)$ using first-order operations (cf. [Mos74]). Moreover, systems of positive first-order formulas provide a normal form for fixpoint logic, i.e., every fixpoint query is definable by the goal $\varphi_{l}^{\infty}$ of some system $\varphi^{1}, \ldots, \varphi^{m}$ of positive first-order formulas.

By the same token, one can also consider extending partial fixpoint logic with systems of arbitrary (not necessarily positive) first-order formulas. If $\varphi_{1}, \ldots, \varphi_{l}$ is a system of first-order formulas, then on every finite structure either there is a stage $\Phi^{m}$ of the operator $\Phi$ such that $\Phi^{m}=\Phi^{m+1}$ or the sequence of stages cycles without ever yielding a fixpoint of $\Phi$. We write $\left(\varphi_{1}^{\infty}, \ldots, \varphi_{l}^{\infty}\right)$ to denote a stage $\Phi^{m}=\left(\Phi_{1}^{m}, \ldots, \Phi_{l}^{m}\right)$ such that $\Phi^{m}=\Phi^{m+1}$, if such a stage exists, or the sequence $(\emptyset, \ldots, \emptyset)$, otherwise. We say that $\left(\varphi_{1}^{\infty}, \ldots, \varphi_{l}^{\infty}\right)$ is the partial fixpoint of the system $\varphi_{1}, \ldots, \varphi_{l}$ and call $\varphi_{l}^{\infty}$ the goal of this system. Using the same simulation as for fixpoint logic, it can be shown that the partial fixpoint rule for systems of first-order formulas does not increase the expressive power of partial fixpoint logic. In addition, every partial fixpoint query is definable by the goal $\varphi_{l}^{\infty}$ of some system of first-order formulas $\varphi_{1}, \ldots, \varphi_{m}$.

In view of the above facts, from now on we extend the syntax of fixpoint logic and partial fixpoint logic by allowing systems of positive first-order formulas and arbitrary first-order formulas, respectively. Using this extended syntax, we can ramify fixpoint logic and partial fixpoint logic into a hierarchy of levels that takes into account the number of variables occurring in systems.

Definition 2.6: Let $\sigma$ be a vocabulary and $k$ a positive integer.

- $\mathrm{FP}^{k}$ is the fragment of fixpoint logic having as formulas the components $\varphi_{i}^{\infty}, 1 \leq i \leq l$, of 
the least fixpoints of systems $\varphi_{1}, \ldots, \varphi_{l}$ of positive first-order formulas over $\sigma$ such that the total number of variables occuring in the systems is at most $k$.

- $\mathrm{PFP}^{k}$ is the fragment of partial fixpoint logic having as formulas the components $\varphi_{i}^{\infty}$, $1 \leq i \leq l$, of the partial fixpoints of systems $\varphi_{1}, \ldots, \varphi_{l}$ of first-order formulas over $\sigma$ such that the total number of variables occuring in the systems is at most $k$.

- If $\mathcal{C}$ is a class of finite structures over the vocabulary $\sigma$, then we write $\mathrm{FP}^{k}(\mathcal{C})$ and $\operatorname{PFP}^{k}(\mathcal{C})$ to denote the class of queries on $\mathcal{C}$ that are definable by formulas of $\mathrm{FP}^{k}$ and $\mathrm{PFP}^{k}$, respectively.

If $\mathcal{C}$ is the class of all finite structures over $\sigma$, then we write simply $\mathrm{FP}^{k}$ and $\mathrm{PFP}^{k}$ to denote the queries on all finite structures that are definable by formulas of $\mathrm{FP}^{k}$ and $\mathrm{PFP}^{k}$, respectively.

According to the above definition, the cycle query is in $\mathrm{FP}^{3}$, since, as seen earlier, it is definable by the component $\varphi_{2}^{\infty}$ of a system of positive first-order formulas with three variables.

In terms of the relationship of fixpoint logic and partial fixpoint logic to complexity classes, it is not hard to verify that

$$
\mathrm{FP} \subseteq \mathrm{PTIME} \text { and } \mathrm{PFP} \subseteq \mathrm{PSPACE}
$$

Moreover, the above containments are proper, since, as shown in [CH82], the even cardinality query, which asks " is there an even number of elements?", is not expressible in PFP. On the other hand, FP and PFP contain queries that are complete respectively for PTIME and PSPACE under logspace reductions and polynomial-time reductions (cf. [CH82, Var82]). In addition, on classes of linearly ordered finite structures fixpoint logic captures PTIME [Imm86, Var82] and partial fixpoint logic captures PSPACE [Var82, AV89]. More formally, if $\sigma$ is a vocabulary containing a binary relation symbol $<$ and $\mathcal{C}$ is a class of finite structures over $\sigma$ such that for every structure $\mathbf{A}$ in $\mathcal{C}$ the relation $<^{\mathbf{A}}$ is a linear order on the universe $A$ of $\mathbf{A}$, then

$$
\operatorname{FP}(\mathcal{C})=\operatorname{PTIME} \text { on } \mathcal{C} \text { and } \operatorname{PFP}(\mathcal{C})=\operatorname{PSPACE} \text { on } \mathcal{C} .
$$

It is clear that $\mathrm{FP} \subseteq \mathrm{PFP}$, since the partial fixpoint of a positive formula coincides with its least fixpoint. Chandra and Harel [CH82] raised the problem of showing that FP is properly contained in PFP on the class of all finite structures over $\sigma$. Abiteboul and Vianu [AV91] established that this separation amounts to separating PTIME from PSPACE, i.e.,

$$
\mathrm{FP}=\mathrm{PFP} \Longleftrightarrow \mathrm{PTIME}=\mathrm{PSPACE} .
$$

The infinitary logic $L_{\infty \omega}^{\omega}$ subsumes both fixpoint logic FP and partial fixpoint logic PFP on finite structures, i.e., FP $\subseteq \mathrm{PFP} \subseteq L_{\infty \omega \omega}^{\omega}$. In fact, as shown in [KV92b, Theorem 2.8], if $\varphi\left(x_{1}, \ldots, x_{l}, S\right)$ is a formula of first-order logic with at most $k$ distinct variables, then the partial fixpoint $\varphi^{\infty}\left(x_{1}, \ldots, x_{l}\right)$ is definable by a formula of $L_{\infty \omega}^{2 k}$. Our first result in the present paper extends this theorem to systems with $k$ variables and at the same time sharpens it to an optimum level by establishing that $k$ variables suffice in transforming formulas of $\mathrm{FP}^{k}$ and $\mathrm{PFP}^{k}$ to formulas of the infinitary logic $L_{\infty \omega}^{\omega}$. More precisely, we will show that $\mathrm{FP}^{k} \subseteq \mathrm{PFP}^{k} \subseteq L_{\infty \omega}^{k}$ for every 
$k \geq 1$. In order to prove this theorem, we need to introduce first an auxiliary concept and obtain an intermediate result. For the remainder of this section we assume that for every $k \geq 1$ the variables $x_{1}, \ldots, x_{k}$ are the $k$ distinct variables of the logics $L_{\omega \omega}^{k}$ and $L_{\infty \omega}^{k}$.

Definition 2.7: Let $k$ be a positive integer and $\pi:\{1, \ldots, k\} \mapsto\{1, \ldots, k\}$ a function. If $Q$ is a $k$-ary query on $\sigma$, then $Q_{\pi}$ is the $k$-ary query on $\sigma$ such that for every finite structure $\mathbf{A}$ over $\sigma$ and every sequence $\left(a_{1}, \ldots, a_{k}\right)$ of elements from the universe $A$ of $\mathbf{A}$

$$
\left(a_{1}, \ldots, a_{k}\right) \in Q_{\pi}(\mathbf{A}) \Longleftrightarrow\left(a_{\pi(1)}, \ldots, a_{\pi(k)}\right) \in Q(\mathbf{A}) .
$$

Lemma 2.8: Let $k$ be a positive integer and $\pi:\{1, \ldots, k\} \mapsto\{1, \ldots, k\}$ be a function. If a $k$-ary query $Q$ on $\sigma$ is $L_{\omega \omega}^{k}$-definable, then the query $Q_{\pi}$ is also $L_{\omega \omega}^{k}$-definable.

Proof: We will show that for every function $\pi:\{1, \ldots, k\} \mapsto\{1, \ldots, k\}$ and for every formula $\varphi\left(x_{1}, \ldots, x_{k}\right)$ of $L_{\omega \omega}^{k}$ there is a formula $\varphi_{\pi}\left(x_{1}, \ldots, x_{k}\right)$ of $L_{\omega \omega}^{k}$ such that for every structure $\mathbf{A}$ over $\sigma$ and every sequence $\left(a_{1}, \ldots, a_{k}\right)$ of elements from the universe of $\mathbf{A}$

$$
\mathbf{A} \models \varphi_{\pi}\left(x_{1} / a_{1}, \ldots, x_{k} / a_{k}\right) \Longleftrightarrow \mathbf{A} \models \varphi\left(x_{1} / a_{\pi(1)}, \ldots, x_{k} / a_{\pi(k)}\right) .
$$

The proof is by induction on the construction of $L_{\omega \omega}^{k}$-formulas simultaneously for all functions $\pi$.

- If $\varphi\left(x_{1}, \ldots, x_{k}\right)$ is the formula $x_{i}=x_{j}$ for some $i, j$ with $1 \leq i \leq j \leq k$, then $\varphi_{\pi}\left(x_{1}, \ldots, x_{k}\right)$ is the formula $x_{\pi(i)}=x_{\pi(j)}$.

- If $R$ is a relation symbol of arity $r$ in the vocabulary $\sigma, q:\{1, \ldots, r\} \mapsto\{1, \ldots, k\}$ is a function, and $\varphi\left(x_{1}, \ldots, x_{k}\right)$ is the atomic formula $R\left(x_{q(1)}, \ldots, x_{q(r)}\right)$, then $\varphi_{\pi}\left(x_{1}, \ldots, x_{k}\right)$ is the formula $R\left(x_{\pi(q(1))}, \ldots, x_{\pi(q(r))}\right)$.

- If $\varphi\left(x_{1}, \ldots, x_{k}\right)$ is a formula of the form $\neg \psi\left(x_{1}, \ldots, x_{k}\right)$, then $\varphi_{\pi}\left(x_{1}, \ldots, x_{k}\right)$ is the formula $\neg \psi_{\pi}\left(x_{1}, \ldots, x_{k}\right)$. Similarly, if $\varphi_{\pi}\left(x_{1}, \ldots, x_{k}\right)$ is of the form $\psi\left(x_{1}, \ldots, x_{k}\right) \wedge \chi\left(x_{1}, \ldots, x_{k}\right)$, then $\varphi_{\pi}\left(x_{1}, \ldots, x_{k}\right)$ is the formula $\psi_{\pi}\left(x_{1}, \ldots, x_{k}\right) \wedge \chi_{\pi}\left(x_{1}, \ldots, x_{k}\right)$.

- Finally, assume that $\varphi\left(x_{1}, \ldots, x_{k}\right)$ is a formula of the form $\left(\exists x_{j}\right) \psi\left(x_{1}, \ldots, x_{k}\right)$ for some $j \leq k$. Let $\pi^{\prime}:\{1, \ldots, k\} \mapsto\{1, \ldots, k\}$ be the function such that $\pi^{\prime}(i)=\pi(i)$, if $i \neq j$, and $\pi^{\prime}(j)=j$. By applying the induction hypothesis to the function $\pi^{\prime}$ and to the formula $\psi\left(x_{1}, \ldots, x_{k}\right)$, we obtain a formula $\psi_{\pi^{\prime}}$ of $L_{\omega \omega}^{k}$ such that for all structures $\mathbf{A}$ over $\sigma$ and all sequences of elements $\left(a_{1}, \ldots, a_{k}\right)$ from the universe of $\mathbf{A}$

$$
\mathbf{A} \models \psi_{\pi^{\prime}}\left(x_{1} / a_{1}, \ldots, x_{k} / a_{k}\right) \Longleftrightarrow \mathbf{A} \models \psi\left(x_{1} / a_{\pi^{\prime}(1)}, \ldots, x_{k} / a_{\pi^{\prime}(k)}\right) .
$$

Then the desired formula $\varphi_{\pi}\left(x_{1}, \ldots, x_{k}\right)$ is the formula $\left(\exists x_{j}\right) \psi_{\pi^{\prime}}\left(x_{1}, \ldots, x_{k}\right)$.

Corollary 2.9: Let $k$ be a positive integer and $\pi:\{1, \ldots, k\} \mapsto\{1, \ldots, k\}$ be a function. If a $k$-ary query $Q$ on $\sigma$ is $L_{\infty \omega}^{k}$-definable, then the query $Q_{\pi}$ is also $L_{\infty \omega}^{k}$-definable. 
We are now ready to show that $\mathrm{PFP}^{k} \subseteq L_{\infty}^{k}$, for every $k \geq 1$, which means that if $\varphi_{1}, \ldots, \varphi_{l}$ is a system of first-order formulas with at most $k$ distinct variables, then each component $\varphi_{i}^{\infty}$ $1 \leq i \leq l$, of the partial fixpoint of the system is definable by a formula of $L_{\infty \omega}^{k}$ on all finite structures.

Theorem 2.10: Let $k, n_{1}, \ldots, n_{l}$ be positive integers such that $n_{i} \leq k$ for every $i \leq l$, let $S_{1}, \ldots S_{l}$ be relation symbols not in the vocabulary $\sigma$ and having arities $n_{1}, \ldots, n_{l}$, and let

$$
\varphi_{1}\left(x_{1}, \ldots, x_{n_{1}}, S_{1}, \ldots, S_{l}\right), \ldots, \varphi_{l}\left(x_{1}, \ldots, x_{n_{l}}, S_{1}, \ldots, S_{l}\right)
$$

be a system of formulas of $L_{\omega \omega}^{k}$ over the vocabulary $\sigma \cup\left\{S_{1}, \ldots, S_{l}\right\}$. Then the following are true for the above system and for the operator $\Phi$ associated with it.

- For every $m \geq 1$, each component $\Phi_{i}^{m}, 1 \leq i \leq l$, of the stage $\Phi^{m}=\left(\Phi_{1}^{m}, \ldots, \Phi_{l}^{m}\right)$ is definable by a formula of $L_{\omega \omega}^{k}$ on all structures (finite or infinite) over $\sigma$.

- Each component $\varphi_{i}^{\infty}, 1 \leq i \leq l$, of the partial fixpoint $\left(\varphi_{1}^{\infty}, \ldots, \varphi_{l}^{\infty}\right)$ of the system is definable by a formula of $L_{\infty \omega}^{k}$ on all finite structures.

Proof: Assume first that $n_{i}=k$ for all $i \leq l$, which means that each $S_{i}$ is a $k$-ary relation symbol not in $\sigma$ and each $\varphi_{i}\left(x_{1}, \ldots, x_{k}, S_{1}, \ldots, S_{l}\right), 1 \leq i \leq k$, is a formula of $L_{\omega \omega}^{k}$ over the vocabulary $\sigma \cup\left\{S_{1}, \ldots, S_{l}\right\}$. By induction on $m$ simultaneously for all $i \leq l$, we will show that each component $\Phi_{i}^{m}$ of every stage $\Phi^{m}$ is definable by a formula $\varphi_{i}^{m}\left(x_{1}, \ldots, x_{k}\right)$ of $L_{\omega \omega}^{k}$. The claim is obvious for $m=1$, since each component $\Phi_{i}^{1}$ of the stage $\Phi^{1}$ is definable by the $L_{\omega \omega}^{k}$ formula $\varphi_{i}\left(x_{1}, \ldots, x_{k}, S_{1} / \emptyset, \ldots, S_{l} / \emptyset\right), 1 \leq i \leq l$. Assume now that there are formulas $\varphi_{i}^{m}\left(x_{1}, \ldots, x_{k}\right)$ of $L_{\omega \omega}^{k}$ that define the components $\Phi_{i}^{m}, 1 \leq i \leq m$ of the stage $\Phi^{m}$, which means that for every structure $\mathbf{A}$ and every sequence $\left(a_{1}, \ldots, a_{k}\right)$ of elements from the universe $A$ of $\mathbf{A}$

$$
\left(a_{1}, \ldots, a_{k}\right) \in \Phi_{i}^{m} \Longleftrightarrow \mathbf{A} \models \varphi_{i}^{m}\left(x_{1} / a_{1}, \ldots, x_{k} / a_{k}\right), 1 \leq i \leq l .
$$

Let us consider the components $\Phi_{i}^{m+1}$ of the stage $\Phi^{m+1}$, which are defined by

$$
\left(a_{1}, \ldots, a_{k}\right) \in \Phi_{i}^{m+1} \Longleftrightarrow \mathbf{A} \models \varphi_{i}\left(x_{1} / a_{1}, \ldots, x_{k} / a_{k}, S_{1} / \Phi_{1}^{m}, \ldots, S_{l} / \Phi_{l}^{m}\right) .
$$

Every occurrence of each relation symbol $S_{j}, 1 \leq j \leq l$, in the formulas of the system is in a subformula of the form $S_{j}\left(x_{\pi(1)}, \ldots, x_{\pi(k)}\right)$ for some function $\pi:\{1, \ldots, k\} \mapsto\{1, \ldots, k\}$. Using the induction hypothesis and applying repeatedly Lemma 2.8, for each $j \leq l$ and each such function $\pi$ we obtain a formula $\varphi_{j, \pi}\left(x_{1}, \ldots, x_{k}\right)$ of $L_{\omega \omega}^{k}$ such that

$$
\left(a_{\pi(1)}, \ldots, a_{\pi(k)}\right) \in \Phi_{j}^{m} \Longleftrightarrow \mathbf{A} \models \varphi_{j, \pi}\left(x_{1} / a_{1}, \ldots, x_{k} / a_{k}\right) .
$$

For every $i \leq l$, let $\varphi_{i}^{m+1}\left(x_{1}, \ldots, x_{k}\right)$ be the formula obtained from $\varphi_{i}\left(x_{1}, \ldots, x_{k}, S_{1}, \ldots, S_{l}\right)$ by replacing each subformula $S_{j}\left(x_{\pi(1)}, \ldots, x_{\pi(k)}\right)$ by the corresponding formula $\varphi_{j, \pi}\left(x_{1}, \ldots, x_{k}\right)$. These replacements can be carried out without renaming variables or introducing new variables. Thus, for every $i \leq m$ the formula $\varphi_{i}^{m+1}\left(x_{1}, \ldots, x_{k}\right)$ is a formula of $L_{\omega \omega}^{k}$ that defines the component $\Phi_{i}^{m}$ of the stage $\Phi^{m+1}$.

Consider next the case that $n_{i}<k$ for at least one $i \leq l$. For every $i \leq l$, let $T_{i}$ be a $k$-ary relation symbol not in the vocabulary $\sigma$ and let $\psi_{i}\left(x_{1}, \ldots, x_{n_{i}}, x_{n_{i}+1}, \ldots, x_{k}, T_{1}, \ldots, T_{l}\right)$ be the 
formula of $L_{\omega \omega}^{k}$ over the vocabulary $\sigma \cup\left\{T_{1}, \ldots, T_{l}\right\}$ obtained from $\varphi_{i}\left(x_{1}, \ldots, x_{n_{i}}, S_{1}, \ldots, S_{l}\right)$ as follows: if $n_{j}<k$, then we replace each subformula $S_{j}\left(x_{\pi(1)}, \ldots, x_{\pi\left(n_{j}\right)}\right)$ by the formula

$$
\left(\exists x_{n_{j}+1}\right) \ldots\left(\exists x_{k}\right) T_{j}\left(x_{\pi(1)}, \ldots, x_{\pi\left(n_{j}\right)}, x_{n_{j}+1}, \ldots, x_{k}\right),
$$

while if $n_{j}=k$, then we replace $S_{j}\left(x_{\pi(1)}, \ldots, x_{\pi\left(n_{j}\right)}\right)$ by $T_{j}\left(x_{\pi(1)}, \ldots, x_{\pi\left(n_{j}\right)}\right)$. A straightforward induction on $m$ simultaneously for all $i \leq m$ shows that if $n_{i}=k$, then $\Phi_{i}^{m}=\Psi_{i}^{m}$, while if $n_{i}<k$, then for every structure $\mathbf{A}$ and every sequence $\left(a_{1}, \ldots, a_{k}\right)$ of elements from the universe of the structure

$$
\left(a_{1}, \ldots, a_{n_{i}}\right) \in \Phi_{i}^{m} \Longleftrightarrow \mathbf{A} \models\left(\exists a_{n_{i}+1} \ldots \exists a_{k}\right)\left(\left(a_{1}, \ldots, a_{n_{i}}, a_{n_{i}+1}, \ldots, a_{k}\right) \in \Psi_{i}^{m}\right) .
$$

For every $m \geq 1$ and $i \leq l$, let $\psi_{i}^{m}\left(x_{1}, \ldots, x_{k}\right)$ be the formula of $L_{\omega \omega}^{k}$ that defines the component $\Psi_{i}^{m}$ of the stage $\Psi^{m}$. If $n_{i}<k$, then we let $\varphi_{i}^{m}\left(x_{1}, \ldots, x_{n_{i}}\right)$ be the formula

$$
\left(\exists x_{n_{i}+1} \ldots \exists x_{k}\right) \psi_{i}^{m}\left(x_{1}, \ldots, x_{n_{i}}, x_{n_{i}+1}, \ldots, x_{k}\right),
$$

while if $n_{i}=k$, then we let $\varphi_{i}^{m}\left(x_{1}, \ldots, x_{k}\right)$ be the formula $\psi_{i}^{m}\left(x_{1}, \ldots, x_{k}\right)$. It follows that $\varphi_{i}^{m}\left(x_{1}, \ldots, x_{n_{i}}\right)$ is a formula of $L_{\omega \omega}^{k}$ that defines the component $\Phi_{i}^{m}$ of the stage $\Phi^{m}, 1 \leq i \leq l$.

Finally, each component $\varphi_{i}^{\infty}\left(x_{1}, \ldots, x_{n_{i}}\right)$ of the partial fixpoint of the system $\varphi_{1}, \ldots, \varphi_{l}$ is definable on all finite structures by the $L_{\infty}^{k}$-formula

$$
\bigvee_{m=1}^{\infty}\left(\bigwedge_{i=i}^{l}\left[\left(\forall x_{1} \ldots \forall x_{n_{i}}\right)\left(\varphi_{i}^{m}\left(x_{1}, \ldots, x_{n_{i}}\right) \leftrightarrow \varphi_{i}^{m+1}\left(x_{1}, \ldots, x_{n_{i}}\right)\right)\right] \wedge \varphi_{i}^{m}\left(x_{1}, \ldots, x_{n_{i}}\right)\right)
$$

Corollary 2.11: For each $k \geq 1, \mathrm{FP}^{k} \subseteq \mathrm{PFP}^{k} \subseteq L_{\infty \omega}^{k}$. As a result, $\mathrm{FP} \subseteq \mathrm{PFP} \subseteq L_{\infty \omega}^{\omega}$.

As mentioned earlier, $L_{\infty \omega}^{\omega}$ on finite graphs can express non-recursive properties. Since every PFP query is in PSPACE, it follows that the inclusion PFP $\subseteq L_{\infty \omega}^{\omega}$ is proper.

\section{Descriptive Complexity of $L_{\infty \omega}^{k}$-Equivalence}

Two structures are $\mathcal{L}$-equivalent in some logic $\mathcal{L}$ if they satisfy the same sentences of $\mathcal{L}$. This is a fundamental concept in the study of every logic, in fact it is as fundamental as the concept of isomorphism in algera. Our goal in this section is to study equivalence in the infinitary $\operatorname{logics} L_{\infty \omega}^{k}$, $k \geq 1$. We begin by reviewing the definitions and stating the known facts about the combinatorial properties of $L_{\infty \omega}^{k}$-equivalence, $k \geq 1$.

Definition 3.1: Let $\mathbf{A}$ and $\mathbf{B}$ be two structures over the vocabulary $\sigma$ and let $k$ be a positive integer.

- We say that $\mathbf{A}$ is $L_{\infty \omega}^{k}$ equivalent to $\mathbf{B}$, and write $\mathbf{A} \equiv_{\infty \omega}^{k} \mathbf{B}$, if $\mathbf{A}$ and $\mathbf{B}$ satisfy the same sentences of $L_{\infty}^{k}$. 
- Let $a_{1}, \ldots, a_{m}$ and $b_{1}, \ldots, b_{m}$ be two sequences of elements from the universes of $\mathbf{A}$ and B respectively, where $1 \leq m \leq k$. We say that $\left(\mathbf{A}, a_{1}, \ldots, a_{m}\right)$ is $L_{\infty \omega}^{k}$-equivalent to $\left(\mathbf{B}, b_{1}, \ldots, b_{m}\right)$, and we write $\left(\mathbf{A}, a_{1}, \ldots, a_{m}\right) \equiv_{\infty \omega}^{k}\left(\mathbf{B}, b_{1}, \ldots, b_{m}\right)$, if for every formula $\varphi\left(x_{1}, \ldots, x_{m}\right)$ of $L_{\infty \omega}^{k}$ with free variables among $x_{1}, \ldots, x_{m}$ we have that

$$
\mathbf{A} \models \varphi\left(x_{1} / a_{1}, \ldots, x_{m} / a_{m}\right) \text { if and only if } \mathbf{B} \models \varphi\left(x_{1} / b_{1}, \ldots, x_{m} / b_{m}\right) .
$$

It is known that the relation $\mathbf{A} \equiv_{\infty \omega}^{k} \mathbf{B}$ can be characterized in terms of an infinitary $k$ pebble game ([Bar77, Imm82]). We define next a variant of this game that captures the relation $\left(\mathbf{A}, a_{1}, \ldots, a_{k}\right) \equiv_{\infty \omega}^{k}\left(\mathbf{B}, b_{1}, \ldots, b_{k}\right)$.

Let $k$ be a positive integer, let $\mathbf{A}$ and $\mathbf{B}$ be two structures over the vocabulary $\sigma$, let $a_{1}, \ldots, a_{k}$ and $b_{1}, \ldots, b_{k}$ be two sequences of elements from the universes of $\mathbf{A}$ and $\mathbf{B}$, respectively, and let $c_{1}^{\mathbf{A}}, \ldots, c_{t}^{\mathbf{A}}$ and $c_{1}^{\mathbf{B}}, \ldots, c_{t}^{\mathbf{B}}$ be the interpretations of the constant symbols $c_{1}, \ldots, c_{t}$ of $\sigma$ on $\mathbf{A}$ and $\mathbf{B}$, respectively. The $k$-pebble game between Players $I$ and II on the structures $\mathbf{A}$ and $\mathbf{B}$ and the sequences $a_{1}, \ldots, a_{k}$ and $b_{1}, \ldots, b_{k}$ has the following rules:

There are $k$ white pebbles $W_{1}, \ldots, W_{k}$ and $k$ black pebbles $B_{1}, \ldots, B_{k}$. Throughout the game, the white pebbles are placed on elements of $\mathbf{A}$ and the black pebbles on elements of $\mathbf{B}$. Initially, the white pebble $W_{j}$ is placed on the element $a_{j}$ of $\mathbf{A}$ and the black pebble $B_{j}$ is placed on the element $b_{j}$ of $\mathbf{B}, 1 \leq j \leq k$. In each round of the game, Player I chooses one of the two colors and moves a pebble of that color. Player II responds by moving the pebble of the other color that has the same index as the pebble moved by Player I.

Let $e_{j}$ and $f_{j}, 1 \leq j \leq k$, be the elements of $\mathbf{A}$ and $\mathbf{B}$ pebbled by $W_{j}$ and $B_{j}$, respectively, at the end of some round of the game. If the mapping $h$ with

$$
h\left(e_{j}\right)=f_{j} \quad \text { and } \quad h\left(c_{j}^{\mathbf{A}}\right)=d_{j}^{\mathbf{B}}, 1 \leq j \leq t,
$$

is not an isomorphism between the substructures of $\mathbf{A}$ and $\mathbf{B}$ with universes $\left\{e_{1}, \ldots, e_{k}\right\} \cup$ $\left\{c_{1}^{\mathbf{A}}, \ldots, c_{l}^{\mathbf{A}}\right\}$ and $\left\{f_{1}, \ldots, f_{k}\right\} \cup\left\{c_{1}^{\mathbf{B}}, \ldots, c_{l}^{\mathbf{B}}\right\}$, respectively, then Player I wins the game. Otherwise, the game continues. Player II wins the game if he can continue playing "forever", i.e. if Player I can never win a round of the game. The $k$-pebble game makes also sense when the two structures $\mathbf{A}$ and $\mathbf{B}$ coincide. In this case, we take two copies of $\mathbf{A}$ and require that the white pebbles are always placed on elements of one copy, while the black pebbles are placed on elements of the other copy. We refer to this game as the $k$-pebble game between Players $I$ and II on the structure $\mathbf{A}$ and the sequences $a_{1}, \ldots, a_{k}$ and $b_{1}, \ldots, b_{k}$.

The crucial connection between $k$-pebble games and $L_{\infty \omega}^{k}$-bequivalence is provided by the following result, which is due to Barwise [Bar77] (cf. also [Imm82]). A detailed proof of this result can be found in $[\mathrm{KV} 90 \mathrm{a}]$.

Theorem 3.2: Let $\mathbf{A}$ and $\mathbf{B}$ be two structures over the vocabulary $\sigma$ and let $k$ be a positive integer. If $a_{1}, \ldots, a_{k}$ and $b_{1}, \ldots, b_{k}$ are sequences of elements from the universes of $\mathbf{A}$ and $\mathbf{B}$, respectively, then the following are equivalent:

1. $\left(\mathbf{A}, a_{1}, \ldots, a_{k}\right) \equiv_{\infty \omega}^{k}\left(\mathbf{B}, b_{1}, \ldots, b_{k}\right)$. 
2. Player II has a winning strategy for the k-pebble game on the structures $\mathbf{A}$ and $\mathbf{B}$ and the sequences $a_{1}, \ldots, a_{k}$ and $b_{1}, \ldots, b_{k}$.

In what follows we shall investigate the descriptive complexity of the query: "given two finite structures $\mathbf{A}$ and $\mathbf{B}$, are they $L_{\infty \omega^{-}}^{k}$ equivalent?", where $k$ is a fixed positive integer. Before stating the main results, however, we need to make the concepts precise.

Let $\sigma$ be a vocabulary and let $\sigma_{1}$ and $\sigma_{2}$ be two disjoint copies of $\sigma$, i.e., for each (relation or constant) symbol $\alpha$ in the vocabulary $\sigma$ we put in $\sigma_{i}$ a symbol $\alpha_{i}$ of the same arity as $\alpha, i=1,2$ (constant symbols are of arity 0 ). We write $\sigma_{1}+\sigma_{2}$ to denote the vocabulary obtained by taking the union of $\sigma_{1}$ and $\sigma_{2}$ and adding two new unary predicate symbols $D_{1}$ and $D_{2}$.

The vocabulary $\sigma_{1}+\sigma_{2}$ is used in order to view pairs of structures over $\sigma$ as single structures over $\sigma_{1}+\sigma_{2}$. Indeed, every pair $(\mathbf{A}, \mathbf{B})$ of structures over $\sigma$ gives rise to a structure $\mathbf{A}+\mathbf{B}$ over $\sigma_{1}+\sigma_{2}$ such that its universe is the union $A \cup B$ of the universes of $\mathbf{A}$ and $\mathbf{B}$, the interpretation of $D_{1}$ (respectively, $D_{2}$ ) is $A$ (respectively, $B$ ), and the interpretation of $\alpha_{1}$ (respectively, $\alpha_{2}$ ) is the interpetation of the symbol $\alpha$ on $\mathbf{A}$ (respectively, $\mathbf{B}$ ). Conversely, we say that a structure $\mathbf{C}$ over $\sigma_{1}+\sigma_{2}$ is proper if its universe is the union of the interpretations of $D_{1}$ and $D_{2}$ and the interpretation of each symbol $\alpha_{i}$ is a relation (or a constant) over the interpretation of $D_{i}, i=1,2$. Thus, out of every proper structure $\mathbf{C}$ we can extract two structures $\left.C\right|_{\sigma_{i}}, i=1,2$, called the restrictions of $\mathbf{C}$, such that $\mathbf{C}=\left.\mathbf{C}\right|_{\sigma_{1}}+\left.\mathbf{C}\right|_{\sigma_{2}}$. It is easy to see that the query "given a structure $\mathbf{C}$ over $\sigma_{1}+\sigma_{2}$, is it a proper structure?" is definable by a first-order sentence over $\sigma_{1}+\sigma_{2}$.

Definition 3.3: Let $\mathcal{L}$ and $\mathcal{L}^{\prime}$ be two logics over the vocabulary $\sigma$.

- We say that $\mathcal{L}$-equivalence of structures is expressible in $\mathcal{L}^{\prime}$ if the following Boolean query is definable by a sentence of $\mathcal{L}^{\prime}$ over $\sigma_{1}+\sigma_{2}$ : "Given a proper finite structure $\mathbf{C}$ over $\sigma_{1}+\sigma_{2}$, is $\left.\mathbf{C}\right|_{\sigma_{1}} \mathcal{L}$-equivalent to $\left.\mathbf{C}\right|_{\sigma_{2}}$ ?"

We refer to this query as the $\mathcal{L}$-equivalence of structures query and to its complement as the $\mathcal{L}$-inequivalence of structures query.

- Let $k$ be a positive integer. We say that $\mathcal{L}$-equivalence of $k$-tuples on two structures is expressible in $\mathcal{L}^{\prime}$ if the following query is definable by a formula of $\mathcal{L}^{\prime}$ over $\sigma_{1}+\sigma_{2}$ : "Given a proper finite structure $\mathbf{C}$ over $\sigma_{1}+\sigma_{2}$ and two sequences of elements $\left(a_{1}, \ldots, a_{k}\right)$ and $\left(b_{1}, \ldots, b_{k}\right)$ from the universes of $\left.\mathbf{C}\right|_{\sigma_{1}}$ and $\left.\mathbf{C}\right|_{\sigma_{2}}$ respectively, does $\left(a_{1}, \ldots, a_{k}\right)$ satisfy on $\left.\mathbf{C}\right|_{\sigma_{1}}$ the same formulas of $\mathcal{L}$ with at most $k$ free variables as $\left(b_{1}, \ldots, b_{k}\right)$ does on $\left.\mathbf{C}\right|_{\sigma_{2}}$ ?"

We refer to this query as the $\mathcal{L}$-equivalence of $k$-tuples on two structure query and to its complement as the the $\mathcal{L}$-inequivalence of $k$-tuples on two structures query.

- Let $k$ be a positive integer. We say that $\mathcal{L}$-equivalence of $k$-tuples on a structure is expressible in $\mathcal{L}^{\prime}$ if the following query is definable by a formula of $\mathcal{L}^{\prime}$ over $\sigma$ : "Given a finite structure A over $\sigma$ and two sequences $\left(a_{1}, \ldots, a_{k}\right)$ and $\left(b_{1}, \ldots, b_{k}\right)$ of elements from $\mathbf{A}$, do they satisfy on $\mathbf{A}$ the same formulas of $\mathcal{L}$ with at most $k$ free variables?"

We refer to this query as the $\mathcal{L}$-equivalence of $k$-tuples on a structure query and to its complement as the the $\mathcal{L}$-inequivalence of $k$-tuples on a structure query.

- In the sequel, we will refer to either one of the previous two queries as the $\mathcal{L}$-equivalence of $k$-tuples query and to its complement as the $\mathcal{L}$-inequivalence of $k$-tuples query. 
For a given logic $\mathcal{L}$, there is no a priori reason for $\mathcal{L}$-equivalence of structures or tuples to be expressible in $\mathcal{L}$ itself. As a matter of fact, one can easily verify that this fails for both first-order logic and the infinitary logic $L_{\infty \omega}^{\omega}$ with a finite number of variables. To see this, notice that, since every finite structure can be described by a first-order sentence up to isomorphism, two finite structures are first-order equivalent if and only if they are isomorphic if and only if they are $L_{\infty \omega}^{\omega}$-equivalent. Towards a contradiction, assume now that there is a sentence $\psi$ of $L_{\infty \omega}^{\omega}$ that defines $L_{\infty \omega}^{\omega}$-equivalence of graphs and let $k$ be the number of distinct variables of $\psi$. It is quite obvious that Player II has a winning strategy for the $k$-pebble game on the complete graphs $K_{k}$ and $K_{k+1}$ with $k$ and $k+1$ nodes, respectively. Thus, by Theorem 3.2, the structure $K_{k}+K_{k+1}$ must satisfy the above sentence $\psi$ and, hence, $K_{k}$ and $K_{k+1}$ must be $L_{\infty \omega}^{\omega}$-equivalent. This, however, is a contradiction, since clearly $K_{k}$ and $K_{k+1}$ can be distinguished by a sentence of first-order logic with $k+1$ variables.

We consider next the infinitary logics $L_{\infty \omega}^{k}, k \geq 1$, and establish lower and upper bounds for the expressibility of $L_{\infty \omega}^{k}$-equivalence.

Proposition 3.4: Let $\sigma$ be a vocabulary containing a binary relation symbol $E$ and let $k$ be a positive integer greater than or equal to 2.

- The $L_{\infty \omega}^{k}$-equivalence of structures query is not expressible in first-order logic.

- The $L_{\infty \omega}^{k}$-equivalence of $k$-tuples query is not expressible in first-order logic.

Proof: Both parts are proved using Ehrenfeucht-Fraïssé games. It is well known (cf. [Fag75]) that for every positive integer $m$ there is an (exponentially bigger) positive integer $M$ such that if $C_{1}=\left(V_{1}, E_{1}\right)$ and $C_{2}=\left(V_{2}, E_{2}\right)$ are two directed cycles each with at least $M$ nodes, then $C_{1}$ and $C_{2}$ satisfy the same first-order sentences of quantifier depth at most $m$. On the other hand, it is easy to see that any two directed cycles of different cardinalities can be distinguished by a sentence of $L_{\infty \omega \omega}^{2}$. Suppose now that the $L_{\infty \omega}^{2}$-equivalence of structures query was expressible by a first-order sentence $\psi$ of quantifier depth $m$. If $C_{1}, C_{2}$ are two directed cycles such that $\left|V_{1}\right| \neq\left|V_{2}\right|$ and $\left|V_{i}\right| \geq M, i=1,2$, then the structure $C_{1}+C_{2}$ must satisfy $\psi$, although $C_{1}$ is not $L_{\infty \omega^{k}}^{k}$-equivalent to $C_{2}$, which is a contradiction.

A variant of this argument can be used to show that the $L_{\infty}^{2}$-equivalence of 2 -tuples on two structures query is not expressible in first-order logic. Let $\mathbf{A}$ be a a directed cycle and let $a_{1}, a_{2}$ be two nodes on this cycle such that the distance from $a_{1}$ to $a_{2}$ is at least $M$. Let $\mathbf{B}$ be a graph that is the disjoint union of two directed cycles $C_{1}$ and $C_{2}$ each with at least $M$ nodes and let $b_{1}, b_{2}$ be two nodes on the cycles $C_{1}$ and $C_{2}$, respectively. It follows that $\left(\mathbf{A}, a_{1}, a_{2}\right)$ and $\left(\mathbf{B}, b_{1}, b_{2}\right)$ satisfy the same first-order formulas of quantifier depth at most $m$, although $\left(\mathbf{A}, a_{1}, a_{2}\right)$ is not $L_{\infty \omega^{-}}^{2}$ equivalent to $\left(\mathbf{B}, b_{1}, b_{2}\right)$.

Using the fact that each of the logics $L_{\infty \omega}^{k}, k \geq 1$, is closed under arbitrary conjunctions, it is not hard to show that they can express their own equivalence.

Proposition 3.5: Let $\sigma$ be a vocabulary and $k$ a positive integer. 
- The $L_{\infty \omega}^{k}$-equivalence of structures query is expressible in $L_{\infty \omega}^{k}$.

- The $L_{\infty \omega^{-}}^{k}$ equivalence of $k$-tuples query is expressible in $L_{\infty \omega}^{2 k}$.

Proof: If $\psi$ is a sentence of $L_{\infty \omega}^{k}$ over the vocabulary $\sigma$, then let $\left.\psi\right|_{\sigma_{i}}$ be the sentence of $L_{\infty \omega}^{k}$ obtained from $\psi$ by replacing each symbol $\alpha$ of $\sigma$ by the symbol $\alpha_{i}$ and relativizing all quantifiers of $\psi$ to the predicates $D_{i}, i=1,2$. The $L_{\infty \omega}^{k}$-equivalence of structures query is expressible by the sentence $\bigwedge_{\psi}\left(\left.\left.\psi\right|_{\sigma_{1}} \rightarrow \psi\right|_{\sigma_{2}}\right)$ of $L_{\infty \omega}^{k}$ over the vocabulary $\sigma_{1}+\sigma_{2}$, where the infinitary conjunction is taken over all sentences $\psi$ of $L_{\infty \omega}^{k}$ over the vocabulary $\sigma$.

The $L_{\infty \omega}^{k}$-equivalence of $k$-tuples on a structure query is expressible by the following formula of $L_{\infty \omega}^{2 k}$ over the vocabulary $\sigma$

$$
\bigwedge_{\varphi}\left(\varphi\left(x_{1}, \ldots, x_{k}\right) \rightarrow\left(\exists x_{1} \ldots \exists x_{k}\right)\left(x_{k+1}=x_{1} \wedge \ldots \wedge x_{2 k}=x_{k} \wedge \varphi\left(x_{1}, \ldots, x_{k}\right)\right)\right),
$$

where the infinitary conjunction is taken over all formulas $\varphi\left(x_{1}, \ldots, x_{k}\right)$ of $L_{\infty}^{k}$ over the vocabulary $\sigma$. In a similar manner, one can find a formula of $L_{\infty \omega}^{2 k}$ over the vocabulary $\sigma_{1}+\sigma_{2}$ that expresses the $L_{\infty \omega}^{k}$-equivalence of $k$-tuples on two structures query.

Since each of the infinitary logics $L_{\infty \omega}^{k}, k \geq 1$, is closed under negations, the preceding Proposition 3.5 implies that the $L_{\infty}^{k}$-inequivalence of structures query is expressible in $L_{\infty \omega}^{k}$ and that the $L_{\infty \omega}^{k}$-inequivalence of $k$-tuples query is expressible in $L_{\infty \omega}^{2 k}$.

In what follows, we establish that both $L_{\infty \omega}^{k}$-equivalence of structures and $L_{\infty \omega^{-}}^{k}$-equivalence of $k$-tuples are also expressible in fixpoint logic. This result depends heavily on the game-theoretic characterization of $L_{\infty}^{k}$-equivalence given in Theorem 3.2.

Theorem 3.6: Let $\sigma$ be a vocabulary and $k$ a positive integer.

- The $L_{\infty \omega}^{k}$-inequivalence of structures query and the $L_{\infty}^{k}$-inequivalence of $k$-tuples query are expressible in $\mathrm{FP}^{2 k}$.

- There is a positive integer $l \geq 2 k$ such that the $L_{\infty \omega^{-}}^{k}$ equivalence of structures query and the $L_{\infty \omega}^{k}$-equivalence of $k$-tuples query are expressible in $\mathrm{FP}^{l}$.

Proof: Let us consider first the $L_{\infty \omega^{k}}^{k}$-inequivalence of $k$-tuples on a structure query. This query asks: "Given a finite structure $\mathbf{A}$ over $\sigma$ and two sequences $\left(a_{1}, \ldots, a_{k}\right)$ and $\left(b_{1}, \ldots, b_{k}\right)$ of elements from $\mathbf{A}$, is $\left(\mathbf{A}, a_{1}, \ldots, a_{k}\right) \not_{\infty \omega}^{k}\left(\mathbf{A}, b_{1} \ldots, b_{k}\right)$ ?". From Theorem 3.2, it follows that this query is equivalent to asking: "Does Player I win the $k$-pebble game on $\mathbf{A}$ and the sequences $a_{1}, \ldots, a_{k}$ and $b_{1}, \ldots, b_{k}$ ?". The latter query is definable by the least fixpoint $\varphi^{\infty}\left(x_{1}, \ldots, x_{k}, x_{k+1}, \ldots, x_{2 k}\right)$ of a positive first-order formula $\varphi\left(x_{1}, \ldots, x_{k}, x_{k+1}, \ldots, x_{2 k}, S\right)$ with a total of $2 k$ distinct variables, which, intuitively, states that Player I wins in the initial configuration or he wins in the "next" move of the game. More precisely, $\varphi\left(x_{1}, \ldots, x_{k}, x_{k+1}, \ldots, x_{2 k}, S\right)$ is the formula

$$
\chi\left(x_{1}, \ldots, x_{k}, x_{k+1}, \ldots, x_{2 k}\right) \vee\left(\bigvee_{i=1}^{k} \psi_{i}\left(x_{1}, \ldots, x_{k}, x_{k+1}, \ldots, x_{2 k}, S\right)\right),
$$

where $\chi$ is a quantifier-free formula stating that the mapping $x_{i} \mapsto x_{k+i}, 1 \leq i \leq k$, is not an isomorphism between the substructures generated by the two tuples $x_{1}, \ldots, x_{k}$ and $x_{k+1}, \ldots, x_{2 k}$, and $\psi_{i}$ is the formula

$$
\left(\exists x_{i}\right)\left(\forall x_{k+i}\right) S\left(x_{1}, \ldots, x_{k}, x_{k+1}, \ldots, x_{2 k}\right) \vee\left(\exists x_{k+i}\right)\left(\forall x_{i}\right) S\left(x_{1}, \ldots, x_{k}, x_{k+1}, \ldots, x_{2 k}\right) .
$$


Using the same reasoning, we can show that the $k$-inequivalence of $k$-tuples query on two structures is expressible in $\mathrm{FP}^{2 k}$, in fact it is definable by the least fixpoint $\theta^{\infty}\left(x_{1}, \ldots, x_{k}, x_{k+1}, \ldots, x_{2 k}\right)$ of a positive first-order formula $\theta\left(x_{1}, \ldots, x_{k}, x_{k+1}, \ldots, x_{2 k}, S\right)$ with $2 k$ distinct variables over the vocabulary $\sigma_{1}+\sigma_{2}$. The only difference is that the formula $\chi$ must be modified appropriately to a formula over the vocabulary $\sigma_{1}+\sigma_{2}$, the quantifiers $\exists x_{i}$ and $\forall x_{i}, 1 \leq i \leq k$, must be relativized to the predicate $D_{1}$, while the quantifiers $\exists x_{k+i}$ and $\forall x_{k+i}, 1 \leq i \leq k$, must be relativized to the predicate $D_{2}$.

It now follows that the $k$-inequivalence of structures query is expressible in $\mathrm{FP}^{2 k}$, since it is definable by the sentence

$$
\begin{array}{r}
\left(\exists x_{1} \ldots \exists x_{k} \forall x_{k+1} \ldots \forall x_{2 k}\right) \theta^{\infty}\left(x_{1}, \ldots, x_{k}, x_{k+1}, \ldots, x_{2 k}\right) \vee \\
\left(\exists x_{k+1} \ldots \exists x_{2 k} \forall x_{1} \ldots \forall x_{k}\right) \theta^{\infty}\left(x_{1}, \ldots, x_{k}, x_{k+1}, \ldots, x_{2 k}\right) .
\end{array}
$$

Finally, by combining the above with Immerman's [Imm86] theorem asserting that queries expressible in fixpoint logic on finite structures are closed under complements, we conclude that the $L_{\infty \omega^{-}}^{k}$-equivalence of structures query and the $L_{\infty \omega}^{k}$-equivalence of $k$-tuples query are expressible in $\mathrm{FP}^{l}$, for some $l \geq 2 k$.

An inspection of Immerman's [Imm86] proof reveals that if a query is expressible by a formula of $\mathrm{FP}^{2 k}$, then its complement is expressible by a formula of $\mathrm{FP}^{8 k}$. In general, an increase in the number of variables needed to express complements of fixpoint queries appears to be necessary (cf. [dR87]). It is an interesting problem to determine if this is indeed the case for the $L_{\infty \omega}^{k}$-equivalence queries.

Question: What is the smallest possible positive integer $l$ such that the query $L_{\infty}^{k}$-equivalence of structures and $L_{\infty \omega}^{k}$-equivalence of $k$-tuples are expressible in $\mathrm{FP}^{l}$ ? In particular, are these queries expressible in $\mathrm{FP}^{2 k}$ ?

It turns out that not only $L_{\infty \omega^{-}}^{k}$-equivalence of structures and $k$-tuples is expressible in fixpoint logic, but that the same holds also for each equivalence class of these equivalence relations.

Definition 3.7: Let $\sigma$ be a vocabulary, $k$ a positive integer, $\mathbf{B}$ a finite structure over $\sigma$, and $w=\left(b_{1}, \ldots, b_{k}\right)$ a sequence of elements from the universe $B$ of $\mathbf{B}$.

- We write $Q_{\mathbf{B}}^{k}$ to denote the $L_{\infty}^{k}$-equivalence class of $\mathbf{B}$ on finite structures, i.e.,

$$
Q_{\mathbf{B}}^{k}=\left\{\mathbf{A}: \mathbf{A} \text { is finite and } \mathbf{A} \equiv_{\infty \omega}^{k} \mathbf{B}\right\} .
$$

Notice that $Q_{\mathbf{B}}^{k}$ can be viewed as a Boolean query over the vocabulary $\sigma$.

- We write $Q_{\mathbf{B}, w}^{k}$ to denote the $L_{\infty \omega^{-}}^{k}$ equivalence class of $\mathbf{B}, w$ on finite structures. Thus, $Q_{\mathbf{B}, w}^{k}$ is the $k$-ary query over $\sigma$ such that, given a finite structure $\mathbf{A}$ over $\sigma$, it returns as value the relation

$$
Q_{\mathbf{B}, w}^{k}(\mathbf{A})=\left\{\left(a_{1}, \ldots, a_{k}\right):\left(\mathbf{A}, a_{1}, \ldots, a_{k}\right) \equiv_{\infty \omega}^{k}\left(\mathbf{B}, b_{1}, \ldots, b_{k}\right)\right\} .
$$

In other words, the query $Q_{\mathbf{B}, w}^{k}$ asks: "Given a finite structure $\mathbf{A}$ over $\sigma$ and a sequence $\left(a_{1}, \ldots, a_{k}\right)$ of elements from the universe $A$ of $\mathbf{A}$, does $\left(\mathbf{A}, a_{1}, \ldots, a_{k}\right) \equiv_{\infty \omega}^{k}\left(\mathbf{B}, b_{1}, \ldots, b_{k}\right)$ ?" 
Theorem 3.8: Let $\sigma$ be a vocabulary, $k$ a positive integer, and $\mathbf{B}$ a finite structure over $\sigma$.

- There is a positive integer $k^{\prime} \geq k$ such that the Boolean query $Q_{\mathrm{B}}^{k}$ is expressible in $\mathrm{FP}^{k^{\prime}}$.

- Let $w_{i}=\left(b_{1}^{i}, \ldots, b_{k}^{i}\right), 1 \leq i \leq l$, be an enumeration of all $k$-tuples of elements from the universe $B$ of $\mathbf{B}$, where $l=|B|^{k}$. There is a system $\varphi_{\mathbf{B}, w_{i}}\left(x_{1}, \ldots, x_{k}, S_{w_{1}}, \ldots, S_{w_{l}}\right), 1 \leq$ $i \leq l$, of positive formulas of $L_{\omega \omega}^{k}$, where $S_{w_{1}}, \ldots, S_{w_{l}}$ are $k$-ary relation symbols not in the vocabulary $\sigma$, such that for every $i \leq l$ the formula $\neg \varphi_{\mathbf{B}, w_{i}}^{\infty}\left(x_{1}, \ldots, x_{k}\right)$ defines the $k$-ary query $Q_{\mathbf{B}, w_{i}}^{k}$, i.e., for every finite structure $\mathbf{A}$ over the vocabulary $\sigma$ and every sequence $\left(a_{1}, \ldots, a_{k}\right)$ of elements from the universe $A$ of $\mathbf{A}$

$$
\left(\mathbf{A}, a_{1}, \ldots, a_{k}\right) \equiv_{\infty \omega}^{k}\left(\mathbf{B}, b_{1}^{i}, \ldots, b_{k}^{i}\right) \Longleftrightarrow \mathbf{A} \models \varphi_{\mathbf{B}, w_{i}}^{\infty}\left(x_{1} / a_{1}, \ldots, x_{k} / a_{k}\right) .
$$

Proof: We begin with the second part of this theorem. For every sequence $w_{i}=\left(b_{1}^{i}, \ldots, b_{k}^{i}\right)$, $1 \leq i \leq l$, of elements from the universe of $\mathbf{B}$, we let $\chi_{\mathbf{B}, w_{i}}\left(x_{1}, \ldots, x_{k}\right)$ be the conjunction of all atomic or negated atomic formulas $\eta\left(x_{1}, \ldots, x_{k}\right)$ such that $\mathbf{B} \models \eta\left(x_{1} / b_{1}^{i}, \ldots, x_{k} / b_{k}^{i}\right)$. Moreover, for every integer $j \leq k$ and every element $b$ from the universe of $\mathbf{B}$, we let $w_{i}[j / b]$ be the $k$-tuple obtained from $w_{i}=\left(b_{1}^{i}, \ldots, b_{k}^{i}\right)$ by replacing $b_{j}^{i}$ by $b$. With this notation at hand, for every $i \leq l$ we let $\varphi_{\mathbf{B}, w_{i}}\left(x_{1}, \ldots, x_{l}, S_{w_{1}} \ldots, S_{w_{l}}\right)$ be the formula

$$
\neg \chi_{\mathbf{B}, w_{i}}\left(x_{1}, \ldots, x_{k}\right) \vee\left[\bigvee_{j=1}^{k}\left(\exists x_{j}\right) \bigwedge_{b \in B} S_{w_{i}[j / b]}\left(x_{1}, \ldots, x_{k}\right)\right] \vee\left[\bigvee_{j=1}^{k} \bigvee_{b \in B}\left(\forall x_{j}\right) S_{w_{i}[j / b]}\left(x_{1}, \ldots, x_{k}\right)\right] .
$$

Note that the above expressions are positive formulas of $L_{\omega \omega}^{k}$ over the vocabulary $\sigma \cup\left\{S_{w_{1}}, \ldots, S_{w_{l}}\right\}$. Using a straightforward induction on $m$ simultaneously for all $i \leq l$, it is not hard to show that for every $m \geq 1$ the components $\Phi_{\mathbf{B}, w_{1}}^{m}, \ldots, \Phi_{\mathbf{B}, w_{l}}^{m}$ of the $m$-th stage of the system $\varphi_{\mathbf{B}, w_{1}}, \ldots, \varphi_{\mathbf{B}, w_{l}}$ possess the following property: for every $i \leq l$, every structure $\mathbf{A}$ over the vocabulary $\sigma$ and every sequence $\left(a_{1}, \ldots, a_{l}\right)$ of elements from the universe of $\mathbf{A}$ we have that $\left(a_{1}, \ldots, a_{k}\right) \in \Phi_{w_{i}}^{m}$ if and only if Player I can win within $m$ rounds the $k$-pebble game on the structures $\mathbf{A}$ and $\mathbf{B}$ and the sequences $\left(a_{1}, \ldots, a_{k}\right)$ and $w_{i}=\left(b_{1}^{i}, \ldots, b_{k}^{i}\right)$. By applying Theorem 3.2 and using the fact that the $k$-pebble game is determined, we conclude that for every $i \leq l$ the component $\varphi_{\mathbf{B}, w_{i}}^{\infty}\left(x_{1}, \ldots, x_{k}\right)$ of the least fixpoint of the system $\varphi_{\mathbf{B}, w_{1}}, \ldots, \varphi_{\mathbf{B}, w_{l}}$ defines the complement of the query $Q_{\mathbf{B}, w_{i}}^{k}$.

It now follows that the Boolean query $Q_{\mathbf{B}}^{k}$ is expressible in fixpoint logic, since its complement is definable by the sentence

$$
\left[\left(\exists x_{1} \ldots \exists x_{k}\right) \bigwedge_{i=1}^{l} \varphi_{\mathbf{B}, w_{i}}^{\infty}\left(x_{1}, \ldots, x_{k}\right)\right] \vee\left[\bigvee_{i=1}^{l}\left(\forall x_{1} \ldots \forall x_{k}\right) \varphi_{\mathbf{B}, w_{i}}^{\infty}\left(x_{1}, \ldots, x_{k}\right)\right]
$$

Dawar, Lindell, and Weinstein [?] established a stronger result than the preceding Theorem 3.8 , namely they showed that for every $k \geq 1$, every finite structure $\mathbf{B}$ over $\sigma$, and every sequence $w=\left(b_{1}, \ldots, b_{k}\right)$ of elements from the universe of $\mathbf{B}$ both the equivalence class $Q_{\mathbf{B}}^{k}$ and the equivalence class $Q_{\mathbf{B}, w}^{k}$ are expressible in $L_{\omega \omega}^{k}$. Dawar et al. [?] proved this result by constructing 
directly $L_{\omega \omega}^{k}$-formulas that define the $L_{\infty \omega}^{k}$-equivalence classes. Their construction is an adaptation of the proof of Scott's theorem [?] to the effect that every countable structure can be described up to isomorphism by a formula of the infinitary logic $L_{\omega_{1} \omega}$. We give next a different proof of the Dawar, Lindell, and Weinstein result, a proof that has game-theoretic flavor and combines Theorem 2.10 with Theorem 3.8.

Theorem 3.9: ([?]) Let $\sigma$ be a vocabulary, $k$ a positive integer, $\mathbf{B}$ a finite structure over $\sigma$, and $w=\left(b_{1}, \ldots, b_{k}\right)$ a sequence of elements from the universe $B$ of $\mathbf{B}$.

- The query $Q_{\mathbf{B}}^{k}$ is definable by a sentence $\theta_{\mathbf{B}}$ of $L_{\omega \omega}^{k}$.

- The query $Q_{\mathbf{B}, w}$ is definable by a formula $\theta_{\mathbf{B}, w}\left(x_{1}, \ldots, x_{k}\right)$ of $L_{\omega \omega}^{k}$.

Proof: Let $w_{i}=\left(b_{1}^{i}, \ldots, b_{k}^{i}\right), 1 \leq i \leq l$, be an enumeration of all $k$-tuples of elements from the universe $B$ of $\mathbf{B}$, where $l=|B|^{k}$. By Theorem 3.8, there is a system $\varphi_{\mathbf{B}, w_{i}}\left(x_{1}, \ldots, x_{k}, S_{w_{1}}, \ldots, S_{w_{l}}\right)$, $1 \leq i \leq l$, of positive formulas of $L_{\omega \omega}^{k}$ such that for every $i \leq l$ the formula $\neg \varphi_{\mathbf{B}, w_{i}}^{\infty}\left(x_{1}, \ldots, x_{k}\right)$ defines the $k$-ary query $Q_{\mathbf{B}, w_{i}}^{k}$. Theorem 2.10 implies that for every $i \leq l$ and every $m \geq 1$ there is a formula $\varphi_{\mathbf{B}, w_{i}}^{m}\left(x_{1}, \ldots, x_{k}\right)$ of $L_{\omega \omega}^{k}$ that defines the component $\Phi_{\mathbf{B}, w_{i}}^{m}$ of the $m$-th stage of this system. Moreover, the proof of Theorem 3.8 reveals that the system $\varphi_{\mathbf{B}, w_{i}}\left(x_{1}, \ldots, x_{k}\right)$, $1 \leq i \leq l$, can be constructed in such a way that for every finite structure $\mathbf{A}$ over $\sigma$ and every sequence $\left(a_{1}, \ldots, a_{k}\right)$ of elements from the universe of $\mathbf{A}$ the following is true: Player I wins within $m$ rounds the $k$-pebble game on the structures $\mathbf{A}$ and $\mathbf{B}$ and the sequences $\left(a_{1}, \ldots, a_{k}\right)$ and $w_{i}=\left(b_{1}^{i}, \ldots, b_{k}^{i}\right)$ if and only if $\mathbf{A} \models \varphi_{\mathbf{B}, w_{i}}^{m}\left(x_{1} / a_{1}, \ldots, x_{k} / a_{k}\right)$. Notice also that for every $m \geq 1$ the restriction of the $k$-pebble game to $m$ rounds constitutes a determined game, which implies that Player II can win the first $m$ rounds the $k$-pebble game on the structures $\mathbf{A}$ and $\mathbf{B}$ and the sequences $\left(a_{1}, \ldots, a_{k}\right)$ and $w_{i}=\left(b_{1}^{i}, \ldots, b_{k}^{i}\right)$ if and only if $\mathbf{A} \models \neg \varphi_{\mathbf{B}, w_{i}}^{m}\left(x_{1} / a_{1}, \ldots, x_{k} / a_{k}\right)$.

Since $\mathbf{B}$ is a finite structure, there is a positive integer $m_{0}$ such that on $\mathbf{B}$ the least fixpoint of the system $\varphi_{\mathbf{B}, w_{i}}\left(x_{1}, \ldots, x_{k}\right), 1 \leq i \leq l$, is equal to its $m_{0}$-th stage, which means that for every $i \leq l$

$$
\mathbf{B} \models\left(\forall x_{1} \ldots \forall x_{k}\right)\left(\varphi_{\mathbf{B}, w_{i}}^{\infty}\left(x_{1}, \ldots, x_{k}\right) \leftrightarrow \varphi_{\mathbf{B}, w_{i}}^{m_{0}}\left(x_{1}, \ldots, x_{k}\right)\right) .
$$

In addition, for every $i \leq l$

$$
\mathbf{B} \models\left(\forall x_{1} \ldots \forall x_{k}\right)\left(\varphi_{\mathbf{B}, w_{i}}^{m_{0}}\left(x_{1}, \ldots, x_{k}\right) \leftrightarrow \varphi_{\mathbf{B}, w_{i}}^{m_{0}+1}\left(x_{1}, \ldots, x_{k}\right)\right) .
$$

We now claim that the query $Q_{\mathbf{B}, w}^{k}$ is definable by the following formula $\theta_{\mathbf{B}, w}\left(x_{1}, \ldots, x_{k}\right)$ of $L_{\omega \omega}^{k}$

$$
\neg \varphi_{\mathbf{B}, w}^{m_{0}}\left(x_{1}, \ldots, x_{k}\right) \wedge\left[\bigwedge_{i=1}^{l}\left(\forall x_{1} \ldots \forall x_{k}\right)\left(\varphi_{\mathbf{B}, w_{i}}^{m_{0}}\left(x_{1}, \ldots, x_{k}\right) \leftrightarrow \varphi_{\mathbf{B}, w_{i}}^{m_{0}+1}\left(x_{1}, \ldots, x_{k}\right)\right)\right] .
$$

It is clear that if $\left(\mathbf{A}, a_{1}, \ldots, a_{k}\right) \equiv_{\infty \omega}^{k}\left(\mathbf{B}, b_{1}, \ldots, b_{k}\right)$, then $\mathbf{A} \models \theta_{\mathbf{B}, w}\left(x_{1} / a_{1}, \ldots, x_{k} / a_{k}\right)$, because $\theta_{\mathbf{B}, w}\left(x_{1}, \ldots, x_{k}\right)$ is a formula of $L_{\omega \omega}^{k}$ satisfied by the structure $\mathbf{B}$ and the sequence $w=\left(b_{1}, \ldots, b_{k}\right)$.

Assume now that $\mathbf{A}$ is a structure over the vocabulary $\sigma$ and $\left(a_{1}, \ldots, a_{k}\right)$ is a sequence of elements from the universe of $\mathbf{A}$ such that $\mathbf{A} \models \theta_{\mathbf{B}, w}\left(x_{1} / a_{1}, \ldots, x_{k} / a_{k}\right)$. We must show that $\left(\mathbf{A}, a_{1}, \ldots, a_{k}\right) \equiv_{\infty \omega}^{k}\left(\mathbf{B}, b_{1}, \ldots, b_{k}\right)$, which, by Theorem 2.10 , amounts to showing that Player II has a winning strategy for the $k$-pebble game on the structures $\mathbf{A}$ and $\mathbf{B}$ and the sequences 
$\left(a_{1}, \ldots, a_{k}\right)$ and $w=\left(b_{1}, \ldots, b_{k}\right)$. We claim that Player II can win the $k$-pebble game by guaranteeing that at any point of time he can win the next $m_{0}$ rounds of the $k$-pebble game. This is certainly true for the starting configuration of the game, since $\mathbf{A} \models \neg \varphi_{w}^{m_{0}}\left(x_{1} / a_{1}, \ldots, x_{k} / a_{k}\right)$ and, as explained earlier, the formula $\neg \varphi_{w}^{m_{0}}\left(x_{1} / a_{1}, \ldots, x_{k} / a_{k}\right)$ is satisfied by a structure $\mathbf{A}$ and a sequence $\left(a_{1}, \ldots, a_{k}\right)$ of elements from the universe of $\mathbf{A}$ if and only if Player II can win the first $m_{0}$ rounds of the $k$-pebble game on the structures $\mathbf{A}$ and $\mathbf{B}$ and the sequences $\left(a_{1}, \ldots, a_{k}\right)$ and $w=\left(b_{1}, \ldots, b_{k}\right)$. Assume that Player II continues to play the $k$-pebble game in such a way that at the end of some round the pebbles have been placed on sequences $\left(a_{1}^{j}, \ldots, a_{k}^{j}\right)$ and $\left(b_{1}^{j}, \ldots, b_{k}^{j}\right)$ having the property that Player II can win the first $m_{0}$ rounds of the $k$-pebble game on the structures $\mathbf{A}$ and $\mathbf{B}$ and the sequences $\left(a_{1}^{j}, \ldots, a_{k}^{j}\right)$ and $w_{j}=\left(b_{1}^{j}, \ldots, b_{k}^{j}\right)$. Thus, $\mathbf{A} \models \neg \varphi_{w_{j}}^{m_{0}}\left(x_{1} / a_{1}^{j}, \ldots, x_{k} / a_{k}^{j}\right)$. Since $\mathbf{A} \models\left(\forall x_{1} \ldots \forall x_{k}\right)\left(\varphi_{w_{j}}^{m_{0}}\left(x_{1}, \ldots, x_{k}\right) \leftrightarrow \varphi_{w_{j}}^{m_{0}+1}\left(x_{1}, \ldots, x_{k}\right)\right)$, it follows that $\mathbf{A} \models \neg \varphi_{w_{j}}^{m_{0}+1}\left(x_{1} / a_{1}^{j}, \ldots, x_{1} / a_{k}^{j}\right)$, which, in turn, implies that Player II can win the first $m+1$ rounds of the $k$-pebble game on the structures $\mathbf{A}$ and $\mathbf{B}$ and the sequences $\left(a_{1}^{j}, \ldots, a_{k}^{j}\right)$ and $w_{j}=\left(b_{1}^{j}, \ldots, b_{k}^{j}\right)$. This makes it possible for Player II to play one more round of the original $k$-pebble game and still guarantee that he can win the subsequent $m$ rounds.

In [KV90a] we proved that every sentence of $L_{\infty \omega}^{k}$ is equivalent to an infinitary disjunction of infinitary conjunctions of $L_{\omega \omega}^{k}$-sentences (i.e., first-order sentences with at most $k$ variables). This result was improved in [KV92a], where it was shown that every formula of $L_{\infty \omega}^{k}$ is equivalent to a countable disjunction of first-order formulas with a fixed number of variables, albeit with more than $k$ variables. Using the preceding Theorem 3.9, Dawar et al. [?] established the following optimal normal form for $L_{\infty \omega}^{k}$.

Theorem 3.10: Let $\sigma$ be a vocabulary and $k$ a positive integer. Every $L_{\infty}^{k}$ formula is equivalent on finite structures to an infinitary disjunction of $L_{\omega \omega}^{k}$-formulas.

Proof: Let $\varphi\left(x_{1}, \ldots, x_{k}\right)$ be a formula of $L_{\infty \omega}^{k}$ over the vocabulary $\sigma$ and let $\mathcal{C}$ be the class of all pairs $(\mathbf{B}, w)$ with the property that $\mathbf{B}$ is a finite structure over $\sigma$ and $w=\left(b_{1}, \ldots, b_{k}\right)$ is a sequence of elements from the universe of $\mathbf{B}$ such that $\left.\mathbf{B} \models \varphi\left(x_{1} / b_{1}\right), \ldots, x_{k} / b_{k}\right)$. Then $\varphi\left(x_{1}, \ldots, x_{k}\right)$ is equivalent on finite structures to the formula

$$
\bigvee_{(\mathbf{B}, w) \in \mathcal{C}} \theta_{\mathbf{B}, w}\left(x_{1}, \ldots, x_{k}\right)
$$

where $\theta_{\mathbf{B}, w}\left(x_{1}, \ldots, x_{k}\right)$ is a formula of $L_{\omega \omega}^{k}$ defining the query $Q_{\mathbf{B}, w}$.

\section{First-Order Logic vs. $L_{\infty \omega}^{\omega}$ and Fixpoint Logic}

\subsection{McColm's Conjectures}

Over the class of all finite structures, $L_{\infty}^{\omega}$ is strictly more expressive than fixpoint logic, and the latter is strictly more expressive than first-order logic. As we observed earlier, however, to fully understand the relative expressive power of these logics, we need to examine their relationship over arbitrary classes of finite structures. On the basis of empirical evidence, it appears that either both fixpoint logic and $L_{\infty \omega}^{\omega}$ are more expressive than first-order logic, e.g., over the class 
of total orders, or both collapse to first-order logic, e.g., over the class of equivalence relations [Kol85].

McColm [McC90] conjectured that the key to the collapse of fixpoint logic and $L_{\infty}^{\omega}$ to firstorder logic is the following boundedness property. Let $\mathcal{C}$ be a class of finite structures over a vocabulary $\sigma$ and let $\varphi(\mathbf{x}, S)$ be a positive first-order formula $\varphi(\mathbf{x}, S)$. We say that the formula $\varphi(\mathbf{x}, S)$ is bounded on $\mathcal{C}$ if there is some $m \geq 1$ such that $\Phi^{m}=\Phi^{\infty}$ over all structures in $\mathcal{C}$. If every positive first-order formula $\varphi(\mathbf{x}, S)$ is bounded on a class $\mathcal{C}$ of finite structures, then we say that fixpoint logic is bounded on $\mathcal{C}$; otherwise, we say that fixpoint logic is unbounded on $\mathcal{C}$.

How is boundedness related to the behavior of fixpoint logic and of $L_{\infty \omega}^{\omega}$ ? It is clear from Theorem 2.10 that if fixpoint logic is bounded on a class $\mathcal{C}$ of finite structures, then fixpoint logic collapses to first-order logic on $\mathcal{C}$. Moreover, McColm [McC90] proved that if fixpoint logic is unbounded on $\mathcal{C}$, then $L_{\infty}^{\omega}$ does not collapse to first-order logic on $\mathcal{C}$. McColm's proof actually shows that if fixpoint logic is unbounded on a class $\mathcal{C}$, then $L_{\infty \omega}^{\omega}$ is strictly more expressive than fixpoint logic on $\mathcal{C}$. In addition, McColm [McC90] observed that the following dichotomy occurs in all known examples: on classes where fixpoint logic and $L_{\infty \omega}^{\omega}$ collapse to first-order logic, fixpoint logic is bounded, while on classes where fixpoint logic and $L_{\infty \omega}^{\omega}$ do not collapse to first-order logic, fixpoint logic is unbounded. These facts led McColm [McC90] to conjecture that boundedness holds the secret to the collapse of fixpoint logic and $L_{\infty \omega}^{\omega}$ to first-order logic.

McColm's First Conjecture: Fixpoint logic collapses to first-order logic on a class $\mathcal{C}$ of finite structures if and only if fixpoint logic is bounded on $\mathcal{C}$.

McColm's Second Conjecture: $L_{\infty \omega}^{\omega}$ collapses to first-order logic on a class $\mathcal{C}$ of finite structures if and only if fixpoint logic is bounded on $\mathcal{C}$.

As explained earlier, the "if" direction of the first conjecture and the "only if" direction of the second one are true. Note also that the two conjectures together imply that $L_{\infty \omega}^{\omega}$ collapses to first-order logic on a class $\mathcal{C}$ of finite structures if and only if fixpoint logic collapses to first-order logic on $\mathcal{C}$.

We now prove McColm's second conjecture, using the game-theoretic characterization of $L_{\infty \omega^{-}}^{k}$ equivalence.

Theorem 4.1: $L_{\infty \omega}^{\omega}$ collapses to first-order logic on a class $\mathcal{C}$ of finite structures if and only if fixpoint logic is bounded on $\mathcal{C}$.

Proof: (Hint) Let $\varphi\left(x_{1}, \ldots, x_{k}, y_{1}, \ldots, y_{k}, S\right)$ be the first-order formula used in the proof of Proposition 3.6 to define $L_{\infty \omega^{-}}^{k}$-inequivalence for tuples, i.e., $\varphi^{\infty}\left(a_{1}, \ldots, a_{k}, b_{1}, \ldots, b_{k}\right)$ holds in a structure $\mathbf{A}$ precisely when $\left(\mathbf{A}, a_{1}, \ldots, a_{k}\right) \not_{\infty \omega}^{k}\left(\mathbf{A}, b_{1}, \ldots, b_{k}\right)$. We can show that there is a function $f$, which depends only on the vocabulary $\sigma$, such that each stage $\varphi^{i}$ of $\varphi$ defines an equivalence relation of index $f(i)$ over any finite structure $\mathbf{A}$ over $\sigma, i \geq 1$. It follows that if fixpoint logic is bounded over a class $\mathcal{C}$ of finite structures, then the number of $L_{\infty \omega^{-}}^{k}$-equivalence classes of tuples is bounded in all structures in $\mathcal{C}$. Using this, we show that each $L_{\infty \omega^{k}}^{k}$-equivalence class of tuples is first-order definable. The result now follows, since each $L_{\infty}^{k} \omega^{\text {-formula defines a }}$ query that is a union of $L_{\infty}^{k}$-equivalence classes. 


\subsection{McColm's First Conjecture: Fixpoint Logic vs. First-order Logic}

McColm's First Conjecture seems to require very different tools than his second conjecture. To gain insight into the problem, we consider a special case by focusing on unbounded classes of ordered structures, i.e., we assume that the vocabulary contains a binary predicate symbol < that is always interpreted as a total order, and, furthermore, we assume that the class contains structures of arbitrarily large (but finite) cardinalities. Without loss of generality, we can assume that the domain of these structures is an initial segment $\{0, \ldots, n-1\}$ of the natural numbers and that $<$ is the restriction to $\{0, \ldots, n-1\}$ of the standard ordering on the natural numbers.

What makes unbounded classes of ordered structures interesting is that the following two important facts hold:

1. Fixpoint logic is unbounded on unbounded classes of ordered structures.

2. Fixpoint logic expresses precisely the polynomial-time properties on unbounded classes of ordered structures.

The first fact is proved easily by exhibiting a fixpoint formula that simply "counts" the number of elements. For example, let $\psi(x, S)$ be the first-order formula $(\forall y)(y<x \rightarrow S(y))$. On ordered structures this formula is unbounded, since $m$-th stage of $\psi$ consists of all numbers in the universe of the structure that are less than or equal to $m-1$. The second fact is a well-known result of [Imm86, Var82].

Thus, on unbounded classes of ordered structures, McColm's First Conjecture becomes:

The Ordered Conjecture: There is no unbounded class of ordered structures on which firstorder logic captures polynomial time.

Note that the Ordered Conjecture can be viewed as a conjecture about the expressive power of first-order logic. It is an open question whether the Ordered Conjecture implies McColm's First Conjecture.

The difficulty in proving the above conjecture comes from the fact that we are dealing with arbitrary classes of finite structures. It is conceivable that the predicates in these structures encode enough information to enable first-order logic to capture polynomial time. Consider, for example, the query $Q(x)$ that returns all even elements in the order <. It is easy to show that this polynomial-time query is not first-order expressible on the class of all ordered structures (the proof uses Ehrenfeucht-Fraïssé games). It is, however, first-order expressible on the classes of ordered structures with a built-in unary predicate $E$ whose interpretation is always the set of even elements in the order $<$. Indeed, the addition of built-in predicates such as the Bit predicate (where $B i t(i, j)$ holds when the $j$-th bit of $i$ is 1 ) is known to significantly increase the expressive power of first-order logic [BIS90, Lin92].

The above discussion makes it clear that to prove the ordered conjecture we have to exhibit a family of polynomial-time queries such that not all of them are expressible in first-order logic on unbounded classes of ordered structures. We now present a family of polynomial-time queries that appears to be such a candidate, on complexity-theoretic grounds.

We first need some technical definitions. For a positive number $n$, let $\operatorname{length}(n)=1+$ $\lfloor\log n\rfloor$. For a nonnegative number $m \leq n$, let $\operatorname{word}_{n}(m)$ be the word comprising of the rightmost length $(n)-1$ bits in the binary representation of $m$. For $k$ nonnegative numbers $m_{1}, \ldots, m_{k} \leq n$, let $\operatorname{word}_{n}\left(m_{1}, \ldots, m_{k}\right)$ be the concatenation $\operatorname{word}_{n}\left(m_{1}\right) \ldots \operatorname{word}_{n}\left(m_{k}\right)$. 
Let $T_{0}, T_{1}, \ldots$ be an enumeration of all exponential-time languages (by exponential we mean $2^{c n}$ for some integer $c>0$ ). For each language $T_{i}$ and each $k>0$, we define a $k$-ary query $Q_{i}^{k}$ as follows: a tuple $\left(m_{1}, \ldots, m_{k}\right)$ is a member of $Q_{i}^{k}(\mathbf{A})$ if and only if $\operatorname{word}_{n}\left(m_{1}, \ldots, m_{k}\right)$ is a member of $T_{i}$, where $n=|\mathbf{A}|$. We denote by $\mathbf{Q}$ the family $\left\{Q_{i}^{k} \mid i \geq 0, k>0\right\}$. It is not hard to see that all queries in $\mathbf{Q}$ are polynomial-time computable.

Our belief in the Ordered Conjecture is supported by the fact that its negation has a certain unlikely complexity-theoretic consequence that will be discussed in detail in the full paper.

Proposition 4.2: Let $\mathcal{C}$ be an unbounded class of ordered structures such that all queries in $\mathbf{Q}$ are expressible in first-order logic over $\mathcal{C}$. Then there is an oracle $A$ with the following property: There is an increasing sequence $n_{1}<n_{2}<\ldots$ such that for every exponential-time language $\mathcal{L}$ and each $k>0$ there is bounded-alternation alternating polynomial-time oracle machine $M$, where, for each $j>0, M^{A}$ can test membership in $\mathcal{L}$ for all strings of length $k n_{j}$, while asking the oracle only questions of length $n_{j}$.

In the full paper, using the techniques of [KL82], we show that if the Ordered Conjecture is false, then one can derive certain complexity-theoretic implications that do not involve an oracle.

\subsection{Ramifying the Conjectures}

McColm's conjectures are stated in terms of first-order logic, fixpoint logics, and $L_{\infty \omega}^{\omega}$. Some of our earlier results (cf. Proposition 3.6, and Theorem 3.10) suggest that these logics are better undersood as union of logics with bounded number of variables, i.e., first-order logic is $\bigcup_{k>0} L_{\omega \omega}^{k}$, fixpoint logic is $\bigcup_{k>0} \mathrm{FP}^{k}$ and $L_{\infty \omega}^{\omega}$ is $\bigcup_{k>0} L_{\infty \omega}^{k}$. It turns out that we can obtain sharper results and formulate finer conjectures if we consider the ramified logics.

We start by considering the logics $L_{\infty \omega}^{k}, k \geq 1$. In the full paper we prove the following proposition, which reveals that $L_{\infty}^{\omega}$ can collapse to first-order only in a ramified manner.

Proposition 4.3: $L_{\infty \omega}^{k}$ collapses to first-order logic over a class $\mathcal{C}$ of finite structures if and only if $L_{\infty \omega}^{k}$ collapses to $L_{\omega \omega}^{k}$ over $\mathcal{C}$.

By combining the above Proposition 4.3 with our earlier Theorem 4.1, we obtain the following stronger solution to McColm's Second Conjecture.

Theorem 4.4: $L_{\infty \omega}^{k}$ collapses to $L_{\omega \omega}^{k}$ on a class $\mathcal{C}$ of finite structures for all $k>0$ if and only if $\mathrm{FP}^{k}$ is bounded on $\mathcal{C}$ for all $k>0$.

This, in turn, suggests considering the ramified version of McColm's First Conjecture.

The Ramified Conjecture: $\mathrm{FP}^{k}$ collapses to $L_{\omega \omega}^{k}$ on a class $\mathcal{C}$ of finite structures for all $k>0$ if and only if $\mathrm{FP}^{k}$ is bounded on $\mathcal{C}$ for all $k>0$.

We conclude by explaining the relationship between the Ramified Conjecture and McColm's First Conjecture. Recall that the (easy) "if" direction of McColm's First Conjecture is a consequence of Theorem 2.10. This theorem, however, does not give the "if" direction of the Ramified Conjecture, because it only implies that if $\mathrm{FP}^{k}$ is bounded on a class $\mathcal{C}$ of finite structures, then $\mathrm{FP}^{k}$ collapses to $L_{\omega \omega}^{2 k}$ on $\mathcal{C}$. We now show that Theorem 2.10 can be sharpened to yield the "if" direction of the Ramified Conjecture. 
Theorem 4.5: Let $\varphi\left(x_{1}, \ldots, x_{k}, S\right)$ be an $L_{\omega \omega}^{k}$ formula over the vocabulary $\sigma \cup\{S\}$ and positive in $S$, where $S$ is a k-ary relation. Then, for every $m \geq 1$, the stage $\Phi^{m}\left(x_{1}, \ldots, x_{k}\right)$ of $\Phi$ is definable by an $L_{\omega \omega}^{k}$-formula on all finite structures over $\sigma$. As a result, the fixpoint $\varphi^{\infty}\left(x_{1}, \ldots, x_{k}\right)$ of $\varphi$ is definable by a formula of $L_{\infty \omega}^{k}$ on all finite structures over $\sigma$.

Corollary 4.6: If $\mathrm{FP}^{k}$ is bounded on a class $\mathcal{C}$ of finite structures for all $k>0$, then $\mathrm{FP}^{k}$ collapses to $L_{\omega \omega}^{k}$ on $\mathcal{C}$ for all $k>0$.

By Corollary 4.6, to prove the Ramified Conjecture it remains to show that if fixpoint logic is not bounded on a class $\mathcal{C}$ of finite structure, then there is some $k>0$ such that $\mathrm{FP}^{k}$ does not collapses to $L_{\omega \omega}^{k}$ on $\mathcal{C}$. Note that to prove McColm's First Conjecture it remains to show the stronger claim that if fixpoint logic is not bounded on a class $\mathcal{C}$ of finite structure, then there is some $k>0$ such that $\mathrm{FP}^{k}$ does not collapses to first-order logic on $\mathcal{C}$.

\subsection{On 0-1 Laws for Infinitary Logics}

Let $\mathcal{C}$ be a class of finite structures and assume that for each $n \geq 1$ we have a probability measure $p r_{n}$ on the collection of all structures in $\mathcal{C}$ with $n$ elements. The asymptotic probability $\operatorname{pr}(P)$ of a property $P$ on $\mathcal{C}$ (relative to the probability measures $\operatorname{pr}_{n}, n \geq 1$ ) is defined as $\operatorname{pr}(P)=\lim _{n \rightarrow \infty}(P)$, provided this limit exists. If $\mathcal{L}$ is a logic, then we say that a $0-1$ law holds for $\mathcal{L}$ on $\mathcal{C}$ relative to the measure $p r$ if for every sentence $\psi$ of $\mathcal{L}$ the asymptotic probability of $\psi$ exists and is either 0 or 1 .

The study of 0-1 laws for various logics has been a major direction of research in finite-model theory (cf. [Com88] for a survey of results). In [KV90a] we showed that the 0-1 law holds for $L_{\infty}^{\omega} \omega$ under the uniform probability measure on the class of all finite structures. There is also a lot of interest in 0-1 laws under variable probability measures and results have been obtained for both first-order logic [SS87] and fixpoint logic [Tys91a, Tys91b]. Moreover, in [KV90a] we found a necessary and sufficient condition for the existence of 0-1 laws for the logics $L_{\infty}^{k}, k \geq 1$, under variable probability measures on arbitrary classes of finite structures. Here, we use the solution to McColm's Second Conjecture to establish a markedly different criterion for the existence of a 0-1 law for the logic $L_{\infty \omega}^{\omega}$.

Theorem 4.7: Let $\mathcal{C}$ be a class of finite structures over a vocabulary $\sigma$ and let $p r_{n}, n \geq 1$, be a sequence of probability measures on the structures of $\mathcal{C}$ with $n$ elements. Then the following are equivalent:

1. The 0-1 law holds for the infinitary logic $L_{\infty}^{\omega}$ on $\mathcal{C}$ relative to the measure pr.

2. The 0-1 law holds for first-order logic on $\mathcal{C}$ relative to the measure $p r$ and for each $k \geq 1$ we have that $\mathrm{FP}^{k}$ is bounded almost everywhere on $\mathcal{C}$, i.e., for each $k \geq 1$ there is a subclass $\mathcal{D}$ of $\mathcal{C}$ such that $\operatorname{pr}(\mathcal{D})=1$ and $\mathrm{FP}^{k}$ is bounded on $\mathcal{D}$.

Proof: (Hint:) Suppose first that the 0-1 law holds for $L_{\infty \omega}^{k}$ relative to the measure $p r$, for some $k>0$. By the results in [KV90a], there is a structure $\mathbf{A}$ in $\mathcal{C}$ such that $\operatorname{pr}([\mathbf{A}])=\mathbf{1}$, where $[\mathbf{A}]$ is the equivalence class of $\mathbf{A}$ with respect to $L_{\infty}^{k}$-equivalence on $\mathcal{C}$. Since $\mathbf{A}$ is a finite structure, there is a positive integer $m_{0}$ such that for every formula $\varphi(\mathbf{x}, S)$ in $L_{\omega \omega}^{k}$ that is positive in $S$ we 
have that $\Phi^{m_{0}}=\Phi^{m_{0}+1}$. For each such $\varphi$, this statement is easily expressible in $L_{\omega \omega}^{k}$ and, as a result it is true on every structure in $[\mathbf{A}]$. Consequently, $\mathrm{FP}^{k}$ is bounded on $[\mathbf{A}]$.

For the other direction, if $\mathrm{FP}^{2 k}$ is bounded on a subclass $\mathcal{D}$ of $\mathcal{C}$, then the proof of Theorem 4.4 shows that $L_{\infty \omega}^{k}$ collapses to $L_{\omega \omega}^{k}$ on $\mathcal{D}$. The $0-1$ law for $L_{\infty \omega}^{k}$ follows now easily from the $0-1$ law for $L_{\omega \omega}^{k}$ and the assumption that $\operatorname{pr}(\mathcal{D})=1$.

In this paper, we solved the second McColm conjecture and brought out the complexitytheoretic character of the first McColm conjecture. In order to preserve the number of open problems, we conclude by offering a conjecture concerning the interplay between 0-1 laws for fixpoint logic and 0-1 laws for $L_{\infty \omega}^{\omega}$.

Conjecture: Let $\mathcal{C}$ be a class of finite structures and let $p r_{n}, n \geq 1$, be a sequence of probability measures on the structures of $\mathcal{C}$ with $n$ elements. Then the 0-1 law holds for the infinitary logic $L_{\infty \omega}^{\omega}$ on $\mathcal{C}$ relative to the measure $p r$ if and only if the $0-1$ law holds for fixpoint logic on $\mathcal{C}$ relative to the measure $p r$.

Acknowledgements: We would like to thank S. Lindell and S. Weinstein for useful discussions and J. Väänänen for useful comments on a previous draft of the paper.

\section{References}

[AG89] M. Ajtai and Y. Gurevich. DATALOG vs. first-order logic. In Proc. 30th IEEE Symp. on Foundations of Computer Science, pages 142-146, 1989.

[Ajt83] M. Ajtai. $\Sigma_{1}^{1}$ formulae on finite structures. Ann. of Pure and Applied Logic, 24:1-48, 1983.

[AU79] A. V. Aho and J. D. Ullman. Universality of data retrieval languages. In Proc. 6th ACM Symp. on Principles of Programming Languages, pages 110-117, 1979.

[AV89] S. Abiteboul and V. Vianu. Fixpoint extensions of first-order logic and Datalog-like languages. In Proc. 4th IEEE Symp. on Logic in Computer Science, pages 71-79, 1989.

[AV91] S. Abiteboul and V. Vianu. Generic computation and its complexity. In Proc. 23rd ACM Symp. on Theory of Computing, pages 209-219, 1991.

[Bar77] J. Barwise. On Moschovakis closure ordinals. Journal of Symbolic Logic, 42:292-296, 1977.

[BF85] J. Barwise and S. Feferman, editors. Model-Theoretic Logics. Springer-Verlag, 1985.

[BIS90] D. A. M. Barrington, N. Immerman, and H. Straubing. On uniformity within NC ${ }^{1}$. Journal of Computer and System Sciences, 41:274-306, 1990.

[CFI89] J. Cai, M. Fürer, and N. Immerman. An optimal lower bound on the number of variables for graph identification. In Proc. 30th IEEE Symp. on Foundations of Computer Science, pages 612-617, 1989. 
[CGKV88] S. S. Cosmadakis, H. Gaifman, P. C. Kanellakis, and M. Y. Vardi. Decidable optimization problems for database logic programs. In Proc. 20th ACM Symp. on Theory of Computing, pages 477-490, 1988.

[CH82] A. Chandra and D. Harel. Structure and complexity of relational queries. Journal of Computer and System Sciences, 25:99-128, 1982.

[Cha88] A. Chandra. Theory of database queries. In Proc. 7th ACM Symp. on Principles of Database Systems, pages 1-9, 1988.

[Com88] K. J. Compton. 0-1 laws in logic and combinatorics. In I. Rival, editor, NATO Adv. Study Inst. on Algorithms and Order, pages 353-383. D. Reidel, 1988.

[dR87] M. de Rougemont. Second-order and inductive definability on finite structures. Zeitschrift für Mathematische Logik und Grundlagen der Mathematik, 33:47-63, 1987.

[Fag75] R. Fagin. Monadic generalized spectra. Zeitschrift für Mathematische Logik und Grundlagen der Mathematik, 21:89-96, 1975.

[Gai82] H. Gaifman. On local and nonlocal properties. In J. Stern, editor, Logic Colloquium '81, pages 105-135. North Holland, 1982.

[GMSV87] H. Gaifman, H. Mairson, Y. Sagiv, and M. Y. Vardi. Undecidable optimization problems for database logic programs. In Proc. 2nd IEEE Symp. on Logic in Computer Science, pages 106-115, 1987.

[GS86] Y. Gurevich and S. Shelah. Fixed-point extensions of first-order logic. Annals of Pure and Applied Logic, 32:265-280, 1986.

[Gur84] Y. Gurevich. Toward logic tailored for computational complexity. In M. M. Ricther et al., editor, Computation and Proof Theory, Lecture Notes in Mathematics 1104, pages 175-216. Springer-Verlag, 1984.

[IL90] N. Immerman and E. S. Lander. Describing graphs: a first-order approach to graph canonization. In A. Selman, editor, Complexity Theory Retrospective, pages 59-81. Springer-Verlag, 1990.

[Imm82] N. Immerman. Upper and lower bounds for first-order expressibility. Journal of Computer and System Sciences, 25:76-98, 1982.

[Imm86] N. Immerman. Relational queries computable in polynomial time. Information and Control, 68:86-104, 1986.

[Ioa86] Y. E. Ioannides. Bounded recursion in deductive databases. Algorithmica, 1:361-385, 1986.

[KA89] P. Kanellakis and S. Abiteboul. Deciding bounded recursion in database logic programs. SIGACT News, 20:4, Fall 1989.

[KL82] R. Karp and R. Lipton. Turing machines that take advice. L' Ensignement Mathématique, 28:191-210, 1982. 
[Kol85] Ph. G. Kolaitis. On asymptotic probabilities of inductive queries and their decision problem. In R. Parikh, editor, Logics of Programs '85, Lecture Notes in Computer Science 193, pages 153-166. Springer-Verlag, 1985.

[KV87] Ph. G. Kolaitis and M. Y. Vardi. The decision problem for the probabilities of higherorder properties. In Proc. 19th ACM Symp. on Theory of Computing, pages 425-435, 1987.

[KV90a] Ph. G. Kolaitis and M. Y. Vardi. 0-1 laws for infinitary logics. In Proc. 5th IEEE Symp. on Logic in Computer Science, pages 156-167, 1990.

[KV90b] Ph. G. Kolaitis and M. Y. Vardi. On the expressive power of Datalog: tools and a case study. In Proc. 9th ACM Symp. on Principles of Database Systems, pages 61-71, 1990. Full version to appear in Journal of Computer and System Sciences. Full version appeared also as IBM Research Report RJ8010, March 1991.

[KV92a] Ph. G. Kolaitis and M. Y. Vardi. Fixpoint logic vs. infinitary logic in finite-model theory. In Proc. Tth IEEE Symp. on Logic in Computer Science, pages 46-57, 1992.

[KV92b] Ph. G. Kolaitis and M. Y. Vardi. Infinitary logic and 0-1 laws. Information and Computation, 98:258-294, 1992. Special issue: Selections from the Fifth Annual IEEE Symposium on Logic in Computer Science.

[Lin92] S. Lindell. The invariant problem for binary string structures and the parallel complexity theory of queries. To appear in J. Computer and System Sciences, 1992.

[LM89] V. S. Lakshmanan and A. O. Mendelzon. Inductive pebble games and the expressive power of DATALOG. In Proc. 8th ACM Symposium on Principles of Database Systems, pages 301-310, 1989.

[McC90] G. L. McColm. When is arithmetics possibe? Annals of Pure and Applied Logic, 50:29-51, 1990 .

[Mos74] Y. N. Moschovakis. Elementary Induction on Abstract Structures. North Holland, 1974 .

[Nau89] J. F. Naughton. Data independent recursion in deductive databases. Journal of Computer and System Sciences, 38:259-289, 1989.

[SS87] J. Spencer and S. Shelah. Threshold spectra for random graphs. In Proc. 19th ACM Symp. on Theory of Computing, pages 421-424, 1987.

[Tys91a] J. Tyszkiewicz. Infinitary queries and their asymptotic probabilities I: properties definable in transitive closure logic. Unpublished Manuscript, 1991.

[Tys91b] J. Tyszkiewicz. Infinitary queries and their asymptotic probabilities II: properties definable in least fixed point logic. Unpublished Manuscript, 1991.

[Var82] M. Y. Vardi. The complexity of relational query languages. In Proc. 14th ACM Symp. on Theory of Computing, pages 137-146, 1982. 